\title{
Local Uniqueness of Ground States for Rotating Bose-Einstein Condensates with Attractive Interactions
}

\author{
Yujin Guo*, Yong $\mathrm{Luo}^{\dagger}$ and Shuangjie Peng
}

December 28, 2021

\begin{abstract}
We study ground states of two-dimensional Bose-Einstein condensates with attractive interactions in a trap $V(x)$ rotating at the velocity $\Omega$. It is known that there exists a critical rotational velocity $0<\Omega^{*}:=\Omega^{*}(V) \leq \infty$ and a critical number $0<a^{*}<\infty$ such that for any rotational velocity $0 \leq \Omega<\Omega^{*}$, ground states exist if and only if the coupling constant $a$ satisfies $a<a^{*}$. For a general class of traps $V(x)$, which may not be symmetric, we prove in this paper that up to a constant phase, there exists a unique ground state as $a \nearrow a^{*}$, where $\Omega \in\left(0, \Omega^{*}\right)$ is fixed.
\end{abstract}

Keywords: Bose-Einstein condensates; rotational velocity; local uniqueness; Pohozaev identity

\section{Introduction}

A Bose-Einstein condensate (BEC) is a state of matter, in which atoms or particles are cooled to the sufficiently low temperature that a large fraction of them "condense" into a single quantum state. Because a BEC can present quantum effects at the macroscopic scale, it has become an important subject in experimental investigations since the first realization 4, 20 of BECs in dilute gases of alkali atoms in 1995. Various interesting quantum phenomena have been observed in the physical experiments of BECs over the past two decades, including the criticalmass collapse [10, 11, 20, 35, 38, 55, the appearance of quantized vortices 1, 9, 14, 23, 57, 59, the center-of-mass rotation [1,23, 48, 57, and so on. These novel experimental progresses promote greatly the developments of mathematical theories and numerical methods arising from BECs, see $1,2,18,20,23,36,37,45,47,57$.

When the interactions between cold atoms in the condensates are repulsive, the quantized vortices and some other complex structures of BECs in rotating traps were analyzed and simulated extensively in the past few years, see review papers [3,46, 54] and the references therein. Due to the distinct mechanism of the critical-mass collapse, the systems of rotating BECs in the attractive case however behave quite different from those of the well-understood repulsive case. For example, vortices are generally unstable in rotating BECs with attractive interactions (see, e.g., 14, 48, 57]), even though vortices are known cf. 2, 17, 23, to form stable lattice configurations in the repulsive case.

\footnotetext{
${ }^{*}$ School of Mathematics and Statistics, and Hubei Key Laboratory of Mathematical Sciences, Central China Normal University, P.O. Box 71010, Wuhan 430079, P. R. China. Email: yguo@mail.ccnu.edu.cn. Y. Guo is partially supported by NSFC under Grant No. 11931012.

${ }^{\dagger}$ School of Mathematics and Statistics, and Hubei Key Laboratory of Mathematical Sciences, Central China Normal University, P.O. Box 71010, Wuhan 430079, P. R. China. Email: yluo@mail.ccnu.edu.cn. Y. Luo is partially supported by the Project funded by China Postdoctoral Science Foundation No. 2019M662680.

${ }^{\ddagger}$ School of Mathematics and Statistics, and Hubei Key Laboratory of Mathematical Sciences, Central China Normal University, P.O. Box 71010, Wuhan 430079, P. R. China. Email: sjpeng@mail.ccnu.edu.cn. S. Peng is partially supported by the Key Project of NSFC under Grant No.11831009.
} 
By a mean-field approximation, see [41, 42, 46, 52, 54, the energy of the two-dimensional attractive BEC in a rotating trap can be described by the following Gross-Pitaevskii (GP) energy functional:

$$
F_{a}(u):=\int_{\mathbb{R}^{2}}\left(|\nabla u|^{2}+V(x)|u|^{2}\right) d x-\frac{a}{2} \int_{\mathbb{R}^{2}}|u|^{4} d x-\Omega \int_{\mathbb{R}^{2}} x^{\perp} \cdot(i u, \nabla u) d x, \quad u \in \mathcal{H},
$$

where $x^{\perp}=\left(-x_{2}, x_{1}\right)$ with $x=\left(x_{1}, x_{2}\right) \in \mathbb{R}^{2},(i u, \nabla u)=i(u \nabla \bar{u}-\bar{u} \nabla u) / 2$, and the complex space $\mathcal{H}$ is defined as

$$
\mathcal{H}:=\left\{u \in H^{1}\left(\mathbb{R}^{2}, \mathbb{C}\right): \int_{\mathbb{R}^{2}} V(x)|u|^{2} d x<\infty\right\} .
$$

Here the parameter $a>0$ in (1.1) characterizes the absolute product of the scattering length $\nu$ of the two-body interaction times the number $N$ of particles in the condensates, and $\Omega \geq 0$ describes the rotational velocity of the rotating trap $V(x) \geq 0$. Following (1.1), ground states of twodimensional attractive BEC in a rotating trap satisfy (cf. [7, 42]) the following mass constraint variational problem:

$$
e_{F}(a):=\inf _{\left\{u \in \mathcal{H},\|u\|_{2}^{2}=1\right\}} F_{a}(u), a>0 .
$$

Alternatively, one may impose $e_{F}(a)$ a different constraint $\int_{\mathbb{R}^{2}}|u(x)|^{2} d x=N>0$, but the latter case can be easily reduced to the previous one with $a$ being replaced by $a / N$. In view of this fact, in this paper we focus on the form of $e_{F}(a)$ instead. We remark that $e_{F}(a)$ is essentially a mass-critical constraint variational problem. The mass-subcritical case of $e_{F}(a)$, where the nonlinear term $|u|^{4}$ is replaced by $|u|^{p}$ for $2<p<4$, was studied as early as in the pioneering work of Esteban-Lions [22].

For the non-rotational case $\Omega=0$ of $e_{F}(a)$, the existence, stability and mass concentration of real-valued minimizers were studied recently in $29,31,33,58$ and the references therein. In this case, it was shown in 29,31,33 that $e_{F}(a)$ admits real-valued minimizers if and only if $a<a^{*}$, where $a^{*}=\|w\|_{L^{2}\left(\mathbb{R}^{2}\right)}^{2}$ and $w=w(|x|)>0$ is the unique (cf. [40, 56]) positive radial solution of the following nonlinear scalar field equation

$$
-\Delta u+u-u^{3}=0 \text { in } \mathbb{R}^{2}, u \in H^{1}\left(\mathbb{R}^{2}, \mathbb{R}\right) .
$$

By the analytical approach of [16, Theorem II.1], this further implies the following existence and nonexistence: for the non-rotational case $\Omega=0, e_{F}(a)$ admits complex-valued minimizers (i.e., ground states) if and only if $a<a^{*}$. Moreover, the mass concentration, symmetry breaking and other analytical properties of minimizers for $e_{F}(a)$ at $\Omega=0$ were analyzed recently for different types of $V(x)$, see $29,31,33,49$ and the references therein.

The rotational case $\Omega>0$ of $e_{F}(a)$ was discussed more recently in [5, 7, 30, 42, where the existence, stability and the limit behavior of complex-valued minimizers were studied. Specially, if the general trapping potential $0 \leq V(x) \in L_{l o c}^{\infty}\left(\mathbb{R}^{2}\right)$ satisfies

$$
\varliminf_{|x| \rightarrow \infty} \frac{V(x)}{|x|^{2}}>0,
$$

the following critical rotational velocity $\Omega^{*}:=\Omega^{*}(V)$ is defined in [30]:

$$
\Omega^{*}:=\sup \left\{\Omega>0: \quad V(x)-\frac{\Omega^{2}}{4}|x|^{2} \rightarrow \infty \text { as }|x| \rightarrow \infty\right\} .
$$

One can note that depending on $V(x)$, both $0<\Omega^{*}<\infty$ and $\Omega^{*}=\infty$ can happen. Under the assumption (1.5), the following existence and non-existence of minimizers for $e_{F}(a)$ were proved in [30, Theorem 1.1]:

Theorem A. Assume $V(x) \in L_{\mathrm{loc}}^{\infty}\left(\mathbb{R}^{2}\right)$ satisfies [1.5) such that $\Omega^{*} \in(0,+\infty]$ in (1.6) exists. Then we have 
1. If $0 \leq \Omega<\Omega^{*}$ and $0 \leq a<a^{*}:=\|w\|_{2}^{2}$, then there exists at least one minimizer of $e_{F}(a)$.

2. If $0 \leq \Omega<\Omega^{*}$ and $a \geq a^{*}:=\|w\|_{2}^{2}$, then there is no minimizer of $e_{F}(a)$.

3. If $\Omega>\Omega^{*}$, then for any $a \geq 0$, there is no minimizer of $e_{F}(a)$.

If $e_{F}(a)$ admits a minimizer $u_{a}$, then the variational theory yields that $u_{a}$ is a ground state of the following Euler-Lagrange equation

$$
-\Delta u_{a}+V(x) u_{a}+i \Omega\left(x^{\perp} \cdot \nabla u_{a}\right)=\mu u_{a}+a\left|u_{a}\right|^{2} u_{a} \quad \text { in } \mathbb{R}^{2},
$$

where $\mu=\mu\left(a, \Omega, u_{a}\right) \in \mathbb{R}$ is a suitable Lagrange multiplier. We remark that the complex-valued solutions of (1.7) were studied directly in [8, 13, 22, 43, and the references therein, where the existence, uniqueness, and other analytical properties of complex-valued solutions were obtained via the Lyapunov-Schmidt reduction, topological degree method, and some other arguments. As for $e_{F}(a)$, suppose $V(x)$ satisfies the assumption (1.5) such that $\Omega^{*} \in(0,+\infty]$ exists, and let $0 \leq \Omega<\Omega^{*}$ be fixed, so that $V_{\Omega}(x):=V(x)-\frac{\Omega^{2}}{4}|x|^{2} \geq 0$ in $\mathbb{R}^{2}$. By deriving energy estimates and applying the elliptic PDE theory, under above assumptions it was proved in 30,42, that the minimizer $u_{a}$ of $e_{F}(a)$ concentrates at a global minimum point of $V_{\Omega}(x)$ as $a \nearrow a^{*}$, in the sense that for some $\varepsilon_{a} \searrow 0$ as $a \nearrow a^{*}$,

$$
\varepsilon_{a} u_{a}\left(\varepsilon_{a}\left(x-x_{0}\right)\right) \rightarrow \frac{w(x)}{\sqrt{a^{*}}} \text { strongly in } H^{1}\left(\mathbb{R}^{2}, \mathbb{C}\right) \text { as } a \nearrow a^{*},
$$

where $x_{0} \in \mathbb{R}^{2}$ is a global minimum point of $V_{\Omega}(x)$, i.e., $V_{\Omega}\left(x_{0}\right):=\inf _{x \in \mathbb{R}^{2}} V_{\Omega}(x)$.

Whether a physics system admits a unique ground state or not is a fundamental and interesting problem. Based on the convergence (1.8), it was further obtained in [30, Theorem 1.3] that the minimizers of $e_{F}(a)$ must be unique and vortex-free as $a \nearrow a^{*}$ for the harmonic case where $V(x)=|x|^{2}$. It deserves emphasis that the conclusions of [30, Theorem 1.3] were proved by the so-called method of inductive symmetry, which cannot however be extended to the non-radially symmetric case of $V(x)$. On the other hand, we should mention that the non-radially symmetric trap $V(x)=|x|^{2}+\left|x_{1}\right|^{2}+\lambda\left|x_{2}\right|^{2}$, where $x=\left(x_{1}, x_{2}\right) \in \mathbb{R}^{2}$ and $\lambda>0$, was already used in BEC experiments, see [50,51] and the references therein. It is therefore natural to wonder whether the uniqueness of minimizers for $e_{F}(a)$ holds for the case where the trap $V(x)$ is not radially symmetric and however satisfies some additional assumptions.

Stimulated by above facts, the main purpose of this paper is to address the uniqueness of minimizers for $e_{F}(a)$ under a more general class of traps $V(x)$, which may not be symmetric. For this purpose, we now introduce the following homogeneous functions:

Definition 1.1. A function $h(x): \mathbb{R}^{2} \longmapsto \mathbb{R}$ is called homogeneous of degree $p \in \mathbb{R}^{+}$(about the origin), if

$$
h(t x)=t^{p} h(x) \text { for any } t \in \mathbb{R}^{+} \text {and } x \in \mathbb{R}^{2} .
$$

One can note that if $\lim _{|x| \rightarrow \infty} h(x)=+\infty$, then $x=0$ is the unique minimum point of $h(x)$. Following the above definition, we next assume that $V(x)$ satisfies

$(V) . V_{\Omega}(x):=V(x)-\frac{\Omega^{2}}{4}|x|^{2} \geq 0$ and $\left\{x \in \mathbb{R}^{2}: V_{\Omega}(x)=0\right\}=\{0\}$, where $V_{\Omega}(x)$ satisfies

$$
\left|V_{\Omega}(x)\right| \leq C e^{\gamma|x|} \text { and }\left|\nabla V_{\Omega}(x)\right| \leq C e^{\gamma|x|} \text { for some } \gamma>0 \text { as }|x| \rightarrow \infty,
$$

and

$$
V_{\Omega}(x)=h(x)+o\left(|x|^{p}\right) \text { and } \frac{\partial V_{\Omega}(x)}{\partial x_{j}}=\frac{\partial h(x)}{\partial x_{j}}+o\left(|x|^{p-1}\right) \text { as }|x| \rightarrow 0, j=1,2
$$

for some homogeneous function $0 \leq h(x) \in C^{1}\left(\mathbb{R}^{2}\right)$ of degree $1<p \leq 2$, where $h(x)$ satisfies $\lim _{|x| \rightarrow \infty} h(x)=+\infty$. 
Example 1.1. Consider the following non-radially symmetric potential

$$
V(x)=|x|^{2}+a_{1}\left|x_{1}\right|^{p}+a_{2}\left|x_{2}\right|^{p}, \quad a_{1} \geq 0, a_{2} \geq 0 \text { and } 1<p \leq 2,
$$

where $x=\left(x_{1}, x_{2}\right) \in \mathbb{R}^{2}$. We remark that when $p=2$, 1.12) gives the harmonic potential considered in [50,51] for BEC experiments. One can easily check that $V(x) \in C_{l o c}^{1, p-1}\left(\mathbb{R}^{2}\right)$ satisfies the assumptions (1.5) and $(V)$ for the homogeneous function $0 \leq h(x) \in C^{1}\left(\mathbb{R}^{2}\right)$ satisfying

$$
\begin{cases}h(x)=a_{1}\left|x_{1}\right|^{p}+a_{2}\left|x_{2}\right|^{p}, & \text { if } 1<p<2 ; \\ h(x)=\left(1-\frac{\Omega^{2}}{4}\right)|x|^{2}+a_{1}\left|x_{1}\right|^{2}+a_{2}\left|x_{2}\right|^{2}, & \text { if } p=2,\end{cases}
$$

where $a_{1} \geq 0$ and $a_{2} \geq 0$ are as in (1.12). Moreover, one can check from (1.13) that

$$
H(y):=\int_{\mathbb{R}^{2}} h(x+y) w^{2}(x) d x
$$

admits a unique critical point $y_{0}=0$, which is also non-degenerate in the sense that

$$
\operatorname{det}\left(\int_{\mathbb{R}^{2}} \frac{\partial h(x)}{\partial x_{j}} \frac{\partial w^{2}(x)}{\partial x_{l}} d x\right)_{j, l=1,2} \neq 0
$$

Under above assumptions, the main result of this paper is concerned with the following local uniqueness.

Theorem 1.1. Suppose $V(x) \in C_{\text {loc }}^{1, \alpha}\left(\mathbb{R}^{2}\right)(0<\alpha<1)$ satisfies 1.5) and $(V)$ for some homogeneous function $0 \leq h(x) \in C^{1}\left(\mathbb{R}^{2}\right)$ of degree $p \in(1,2]$, and let $\Omega \in\left(0, \Omega^{*}\right)$ be fixed, where $\Omega^{*}>0$ is defined as in (1.6). Assume that $H(y)$ defined by (1.14) has a unique non-degenerate critical point $y_{0}$, then up to a constant phase, there exists a unique complex-valued minimizer of $e_{F}(a)$ for $a^{*}-a>0$ small enough.

We remark that the local uniqueness, up to a constant phase, of Theorem 1.1 holds in the following sense: there exists a minimizer $u_{a}$ of $e_{F}(a)$ such that any minimizer $U_{a}$ of $e_{F}(a)$ satisfies $U_{a} \equiv u_{a} e^{i \theta_{a}}$ in $\mathbb{R}^{2}$ for $a^{*}-a>0$ small enough, where $\theta_{a} \in[0,2 \pi)$ is a suitable constant phase depending on $a$. We note that depending on the shape of $h(x)$, the unique non-degenerate critical point of $H(y)$ can be nonzero. Example1.1 gives an explicit example that the uniqueness of Theorem 1.1 holds for non-radially symmetric traps $V(x)$.

Even though the similar local uniqueness of complex-valued solutions was investigated in 13.30 and somewhere else, to our best knowledge, those arguments do not work in our situation. Actually, it seems that the topological degree argument (e.g. 13]) does not work for proving Theorem 1.1, due to the possible multiplicity of the Lagrange multiplier $\mu=\mu(a) \in \mathbb{R}$ in the following Euler-Lagrange equation

$$
-\Delta u_{a}+V(x) u_{a}+i \Omega\left(x^{\perp} \cdot \nabla u_{a}\right)=\mu u_{a}+a\left|u_{a}\right|^{2} u_{a} \text { in } \mathbb{R}^{2}
$$

for any given $0<a<a^{*}$. On the other hand, the local uniqueness of [30, Theorem 1.3] follows strongly from the conclusion that if $V(x)=|x|^{2}$, then $\operatorname{Im}\left(u_{a}\right) \equiv 0$ as $a \nearrow a^{*}$, which is however false generally for the non-radially symmetric case of $V(x)$. Therefore, it is necessary to investigate a different approach for proving Theorem [1.1. Motivated by [12, 21, 27, 29] and the references therein, we shall prove Theorem 1.1 by constructing various Pohozaev identities, which were widely used in the existing literature of studying the real-valued elliptic PDEs.

To illustrate our main idea of proving Theorem 1.1. by contradiction we now suppose that up to a constant phase, there exist two different minimizers $u_{1, a}$ and $u_{2, a}$ of $e_{F}(a)$ as $a \nearrow a^{*}$, in the sense that $u_{1, a} \not \equiv u_{2, a} e^{i \theta}$ for any constant phase $\theta=\theta(a) \in[0,2 \pi)$. Motivated by [12, 21, 27, 30], we make the following transformation of $u_{j, a}$ :

$$
\tilde{u}_{j, a}(x):=\alpha_{a} u_{j, a}\left(\alpha_{a}\left(x+y_{0}\right)\right) e^{-i\left(\frac{\alpha_{a}^{2} \Omega}{2} x \cdot y_{0}^{\perp}\right)} e^{i \varphi_{j, a}}=R_{j, a}(x)+i I_{j, a}(x), \quad j=1,2,
$$


where $\alpha_{a}:=\frac{\left(a^{*}-a\right)^{\frac{1}{2+p}}}{\lambda}>0$ is given in (2.12), the point $y_{0} \in \mathbb{R}^{2}$ is as in Theorem 1.1] and the constant phase $\varphi_{j, a} \in[0,2 \pi)$ can be chosen properly such that

$$
\int_{\mathbb{R}^{2}} w(x) I_{j, a}(x) d x=0, \quad j=1,2 .
$$

Under the assumptions of Theorem 1.1, we shall prove in Proposition 2.2 that $\tilde{u}_{j, a}(x)$ satisfies

$$
\lim _{a \nearrow a^{*}} \tilde{u}_{j, a}(x)=\frac{w(x)}{\sqrt{a^{*}}} \text { strongly in } L^{\infty}\left(\mathbb{R}^{2}, \mathbb{C}\right) \cap H^{1}\left(\mathbb{R}^{2}, \mathbb{C}\right), \quad j=1,2,
$$

which is the first step of proving Theorem 1.1

Because $u_{1, a} \not \equiv u_{2, a} e^{i \theta}$ for any constant phase $\theta=\theta(a) \in[0,2 \pi)$, we next define

$$
\eta_{a}(x):=\frac{\tilde{u}_{2, a}(x)-\tilde{u}_{1, a}(x)}{\left\|\tilde{u}_{2, a}-\tilde{u}_{1, a}\right\|_{L^{\infty}\left(\mathbb{R}^{2}\right)}}=\eta_{1, a}(x)+i \eta_{2, a}(x),
$$

where $\eta_{1, a}$ and $\eta_{2, a}$ denote the real and imaginary parts of $\eta_{a}$, respectively. In order to continue the proof of Theorem 1.1 the second step is to establish the refined $L^{\infty}$-uniform estimates of both $\eta_{1, a}$ and $\eta_{2, a}$ as $a \nearrow a^{*}$. By making full use of (1.18), we shall reach this aim by proving Lemma 3.3 and Proposition 3.4, which reveal that $\eta_{1, a}$ is the dominant part of $\eta_{a}(x)$ as $a \nearrow a^{*}$. As a result, we shall be able to prove that up to a subsequence if necessary, $\left(\eta_{1, a}, \eta_{2, a}\right) \rightarrow\left(\eta_{1}, \eta_{2}\right)$ uniformly in $L^{\infty}\left(\mathbb{R}^{2}\right)$ as $a \nearrow a^{*}$, where $\left(\eta_{1}, \eta_{2}\right)$ satisfies the following system

$$
\left\{\begin{array}{l}
-\Delta \eta_{1}+\eta_{1}-3 w^{2} \eta_{1}=-\left(\frac{2}{a^{*}} \int_{\mathbb{R}^{2}} w^{3} \eta_{1} d x\right) w \text { in } \mathbb{R}^{2}, \\
-\Delta \eta_{2}+\eta_{2}-w^{2} \eta_{2}=0 \text { in } \mathbb{R}^{2}, \int_{\mathbb{R}^{2}} w \eta_{2} d x=0,
\end{array}\right.
$$

and hence

$$
\eta_{1}=b_{0}(w+x \cdot \nabla w)+\sum_{i=1}^{2} b_{i} \frac{\partial w}{\partial x_{i}} \quad \text { and } \quad \eta_{2} \equiv 0 \text { in } \mathbb{R}^{2}
$$

for some constants $b_{0}, b_{1}$ and $b_{2}$.

In the third step of proving Theorem 1.1, we shall prove that $b_{0}=b_{1}=b_{2}=0$ holds for (1.22) by constructing various Pohozaev identities. Due to the appearance of the rotating term $i\left(x^{\perp} \cdot \nabla \tilde{u}_{j, a}\right)$, it however seems difficult to establish directly Pohozaev identities of the complexvalued function $\tilde{u}_{j, a}$. To overcome this difficulty, as illustrated before (4.5), we shall construct various Pohozaev identities for the real part $R_{j, a}$ of $\tilde{u}_{j, a}:=R_{j, a}+i I_{j, a}$. A key point of this strategy is to derive the refined estimates for the terms produced by the rotation. Applying Lemma 3.1 and Proposition 3.4, we shall prove that those terms produced by the rotation are lower order as $a \nearrow a^{*}$, based on which we shall be able to establish the system (4.5) concerning the relationship between the homogeneous potential $h(x)$ and the coefficients $b_{0}, b_{1}$ and $b_{2}$. By deriving another type of Pohozaev identities, we shall further prove that $b_{0}=0$. Following this fact and the non-degeneracy assumption of $y_{0}$, we shall derive from (4.5) that $b_{1}=b_{2}=0$ holds for (1.22), and hence,

$$
\eta_{a}=\eta_{1, a}+i \eta_{2, a} \rightarrow \eta_{0}=\eta_{1}+i \eta_{2} \equiv 0 \text { uniformly in } L^{\infty}\left(\mathbb{R}^{2}\right) \text { as a } \nearrow a^{*} .
$$

On the other hand, one can conclude from Lemma 3.3 that $\eta_{0}=\eta_{1}+i \eta_{2} \not \equiv 0$ in view of the fact that $\left\|\eta_{a}\right\|_{L^{\infty}} \equiv 1$. This is a contradiction, and Theorem 1.1 therefore follows.

This paper is organized as follows. In Section 2, we shall analyze the $L^{\infty}$-uniform estimates of minimizers for $e_{F}(a)$ as $a \nearrow a^{*}$. In Section 3, we shall first derive a crucial gradient estimate of (3.10), based on which the refined limit profiles of minimizers as $a \nearrow a^{*}$ are then established in Lemma 3.3 and Proposition 3.4 Following the estimates of Section 3, the complete proof of Theorem 1.1 is finally addressed in Section 4 by constructing various Pohozaev identities. 


\section{$2 \quad L^{\infty}$-uniform estimates as $a \nearrow a^{*}$}

The purpose of this section is to address $L^{\infty}$-uniform estimates of the complex-valued minimizers for $e_{F}(a)$ as $a \nearrow a^{*}$. Towards this purpose, we first introduce the following Gagliardo-Nirenberg inequality

$$
\int_{\mathbb{R}^{2}}|u(x)|^{4} d x \leq \frac{2}{a^{*}} \int_{\mathbb{R}^{2}}|\nabla u(x)|^{2} d x \int_{\mathbb{R}^{2}}|u(x)|^{2} d x, \quad u \in H^{1}\left(\mathbb{R}^{2}, \mathbb{R}\right),
$$

where the equality is attained (cf. [56]) at the unique positive radial solution $w$ of (1.4). Moreover, it follows from [15, Lemma 8.1.2] that $w=w(|x|)>0$ satisfies

$$
\int_{\mathbb{R}^{2}}|\nabla w|^{2} d x=\int_{\mathbb{R}^{2}} w^{2} d x=\frac{1}{2} \int_{\mathbb{R}^{2}} w^{4} d x
$$

and note from [25, Proposition 4.1] that

$$
w(x),|\nabla w(x)|=O\left(|x|^{-\frac{1}{2}} e^{-|x|}\right) \quad \text { as }|x| \rightarrow \infty .
$$

Given any vector function $\mathcal{A} \in L_{\text {loc }}^{2}\left(\mathbb{R}^{2}, \mathbb{R}^{2}\right)$, recall also from 44 the following diamagnetic inequality:

$$
|(\nabla-i \mathcal{A}) u|^{2} \geq|\nabla| u||^{2} \text { a.e. on } \mathbb{R}^{2}, u \in H^{1}\left(\mathbb{R}^{2}, \mathbb{C}\right) .
$$

In this paper, we often use the following linearized operator

$$
\mathcal{L}:=-\Delta+1-w^{2} \quad \text { in } \mathbb{R}^{2} .
$$

It then obtains from [24] that

$$
\operatorname{ker} \mathcal{L}=\{w\} \quad \text { and }\langle\mathcal{L} v, v\rangle \geq 0 \text { for all } v \in L^{2}\left(\mathbb{R}^{2}\right),
$$

see also [44, Theorem 11.8] and [44, Corollary 11.9]. Further, by a standard argument (e.g. (3.45) in [30), there exists $\rho>0$ such that

$$
\langle\mathcal{L} v, v\rangle \geq \rho\|v\|_{H^{1}\left(\mathbb{R}^{2}\right)}^{2} \text { for all } v \in \mathcal{S},
$$

where the space $\mathcal{S}$ is defined as

$$
\mathcal{S}:=\left\{v \in H^{1}\left(\mathbb{R}^{2}, \mathbb{R}\right): \int_{\mathbb{R}^{2}} w(x) v(x) d x=0\right\} .
$$

Denote the linearized operator $\mathcal{N}$ by

$$
\mathcal{N}:=-\Delta+1-3 w^{2} \quad \text { in } \mathbb{R}^{2} .
$$

It then follows from [24,40,53, that

$$
\operatorname{ker} \mathcal{N}=\operatorname{span}\left\{\frac{\partial w}{\partial x_{1}}, \frac{\partial w}{\partial x_{2}}\right\}
$$

Here and in the sequel, we always denote $u_{a}$ to be a complex-valued minimizer of $e_{F}(a)$, where the rotating speed $\Omega \in\left(0, \Omega^{*}\right)$ is fixed. By the variational theory, there exists a Lagrange multiplier $\mu_{a} \in \mathbb{R}$ satisfying

$$
\mu_{a}=e_{F}(a)-\frac{a}{2} \int_{\mathbb{R}^{2}}\left|u_{a}\right|^{4} d x
$$

such that $u_{a}$ solves the following Euler-Lagrange equation:

$$
-\Delta u_{a}+V(x) u_{a}+i \Omega\left(x^{\perp} \cdot \nabla u_{a}\right)=\mu_{a} u_{a}+a\left|u_{a}\right|^{2} u_{a} \text { in } \mathbb{R}^{2} .
$$


Under the assumptions of Theorem 1.1, we also define

$$
\alpha_{a}:=\frac{\left(a^{*}-a\right)^{\frac{1}{2+p}}}{\lambda}>0, \text { where } 1<p \leq 2,
$$

and

$$
\lambda= \begin{cases}{\left[\frac{p}{2} \int_{\mathbb{R}^{2}} h\left(x+y_{0}\right) w^{2}(x) d x\right]^{\frac{1}{2+p}},} & \text { if } \quad 1<p<2 ; \\ {\left[\int_{\mathbb{R}^{2}}\left(h\left(x+y_{0}\right)+\frac{\Omega^{2}}{4}|x|^{2}\right) w^{2}(x) d x\right]^{\frac{1}{4}},} & \text { if } \quad p=2,\end{cases}
$$

where $y_{0} \in \mathbb{R}^{2}$ denotes the unique non-degenerate critical point of $H(y)$, see Theorem 1.1 Setting

$$
\varepsilon_{a}:=\left(\int_{\mathbb{R}^{2}}\left|\nabla u_{a}\right|^{2} d x\right)^{-\frac{1}{2}}>0,
$$

we now define

$$
w_{a}(x):=\varepsilon_{a} u_{a}\left(\varepsilon_{a} x+x_{a}\right) e^{-i\left(\frac{\varepsilon_{a} \Omega}{2} x \cdot x_{a}^{\perp}-\theta_{a}\right)},
$$

where $x_{a}$ is a global maximal point of $\left|u_{a}(x)\right|$ and $\theta_{a} \in[0,2 \pi)$ is a proper constant. Using above notations, since the proof of the following lemma is similar to those of [30. Section 3], we omit the details of the proof for simplicity.

Lemma 2.1. Under the assumptions of Theorem 1.1, let $u_{a}$ be a minimizer of $e_{F}(a)$. Then we have

(i). The parameter $\varepsilon_{a}>0$ satisfies

$$
\varepsilon_{a}=\alpha_{a}+o\left(\alpha_{a}\right)>0 \text { and } \mu_{a} \varepsilon_{a}^{2} \rightarrow-1 \text { as a } \nearrow a^{*},
$$

where $\mu_{a} \in \mathbb{R}$ is the Lagrange multiplier of (2.11).

(ii). The function $w_{a}(x)$, defined in (2.15) for some suitable constant $\theta_{a} \in[0,2 \pi)$, satisfies

$$
\lim _{a \nearrow a^{*}} w_{a}(x)=\frac{w(x)}{\sqrt{a^{*}}} \text { strongly in } L^{\infty}\left(\mathbb{R}^{2}, \mathbb{C}\right) \cap H^{1}\left(\mathbb{R}^{2}, \mathbb{C}\right) \text { as a } \nearrow a^{*},
$$

and there exists a constant $C>0$, independent of $0<a<a^{*}$, such that $w_{a}(x)$ satisfies

$$
\left|w_{a}(x)\right| \leq C e^{-\frac{2}{3}|x|} \quad \text { uniformly in } \mathbb{R}^{2} \text { as a } \nearrow a^{*} .
$$

(iii). The global maximal point $x_{a}$ of $\left|u_{a}\right|$ must be unique as a $\nearrow a^{*}$, and $x_{a}$ satisfies

$$
\lim _{a \nearrow a^{*}} \frac{x_{a}}{\varepsilon_{a}}=\lim _{a \nearrow a^{*}} \frac{x_{a}}{\alpha_{a}}=y_{0},
$$

where $y_{0} \in \mathbb{R}^{2}$ denotes the unique non-degenerate critical point of $H(y)$.

Applying Lemma 2.1, we now consider

$$
v_{a}(x):=\alpha_{a} u_{a}\left(\alpha_{a}\left(x+y_{0}\right)\right) e^{-i\left(\frac{\Omega \alpha_{a}^{2}}{2} x \cdot y_{0}^{\perp}\right)} e^{i \varphi_{a}}=R_{a}(x)+i I_{a}(x),
$$

where $R_{a}(x)$ and $I_{a}(x)$ denote the real and imaginary parts of $v_{a}(x)$, respectively, and the constant phase $\varphi_{a} \in[0,2 \pi)$ is chosen such that

$$
\left\|v_{a}-\frac{w}{\sqrt{a^{*}}}\right\|_{L^{2}\left(\mathbb{R}^{2}\right)}=\min _{\theta \in[0,2 \pi)}\left\|e^{i \theta} v_{a}-\frac{w}{\sqrt{a^{*}}}\right\|_{L^{2}\left(\mathbb{R}^{2}\right)} .
$$

This gives the following orthogonality condition on $I_{a}(x)$, which plays an essential role in proving Theorem 1.1

$$
\int_{\mathbb{R}^{2}} w(x) I_{a}(x) d x=0 .
$$

Based on Lemma 2.1, we next derive the following $L^{\infty}$-uniform estimates of $v_{a}$ : 
Proposition 2.2. Under the assumptions of Theorem 1.1, assume $v_{a}(x)$ and $\varphi_{a} \in[0,2 \pi)$ are defined by (2.20) and (2.21), respectively. Then we have

(i). The function $v_{a}(x)$ satisfies

$$
\lim _{a \nearrow a^{*}} v_{a}(x)=\frac{w(x)}{\sqrt{a^{*}}} \text { strongly in } L^{\infty}\left(\mathbb{R}^{2}, \mathbb{C}\right) \cap H^{1}\left(\mathbb{R}^{2}, \mathbb{C}\right) .
$$

(ii). There exists a constant $C>0$ such that

$$
\left|v_{a}(x)\right| \leq C e^{-\frac{2}{3}|x|}, \quad\left|\nabla v_{a}(x)\right| \leq C e^{-\frac{1}{2}|x|} \text { in } \mathbb{R}^{2} \text { as a } \nearrow a^{*} .
$$

Proof. 1. We first derive from (2.16), (2.17) and (2.19) that

$$
\lim _{a \nearrow a^{*}} \alpha_{a} u_{a}\left(\alpha_{a}\left(x+y_{0}\right)\right) e^{-i\left(\frac{\Omega \alpha_{a}^{2}}{2} x \cdot y_{0}^{\perp}\right)} e^{i \theta_{a}}=\frac{w(x)}{\sqrt{a^{*}}} \text { strongly in } L^{\infty}\left(\mathbb{R}^{2}, \mathbb{C}\right) \cap H^{1}\left(\mathbb{R}^{2}, \mathbb{C}\right),
$$

where $\theta_{a} \in[0,2 \pi)$ is defined by (2.15) such that (2.17) holds. We then obtain from (2.25) that

$$
\begin{aligned}
& \lim _{a \nearrow a^{*}}\left\|\alpha_{a} u_{a}\left(\alpha_{a}\left(x+y_{0}\right)\right) e^{-i\left(\frac{\Omega \alpha_{a}^{2}}{2} x \cdot y_{0}^{\perp}\right)}\left(e^{i \theta_{a}}-e^{i \varphi_{a}}\right)\right\|_{L^{2}\left(\mathbb{R}^{2}\right)} \\
\leq & \lim _{a \nearrow a^{*}}\left\|\alpha_{a} u_{a}\left(\alpha_{a}\left(x+y_{0}\right)\right) e^{-i\left(\frac{\Omega \alpha_{a}^{2}}{2} x \cdot y_{0}^{\perp}\right)} e^{i \theta_{a}}-\frac{w}{\sqrt{a^{*}}}\right\|_{L^{2}\left(\mathbb{R}^{2}\right)} \\
+ & \lim _{a \nearrow a^{*}}\left\|\alpha_{a} u_{a}\left(\alpha_{a}\left(x+y_{0}\right)\right) e^{-i\left(\frac{\Omega \alpha_{a}^{2}}{2} x \cdot y_{0}^{\perp}\right)} e^{i \varphi_{a}}-\frac{w}{\sqrt{a^{*}}}\right\|_{L^{2}\left(\mathbb{R}^{2}\right)} \\
\leq & \lim _{a \nearrow a^{*}} 2\left\|\alpha_{a} u_{a}\left(\alpha_{a}\left(x+y_{0}\right)\right) e^{-i\left(\frac{\Omega \alpha_{a}^{2}}{2} x \cdot y_{0}^{\perp}\right)} e^{i \theta_{a}}-\frac{w}{\sqrt{a^{*}}}\right\|_{L^{2}\left(\mathbb{R}^{2}\right)}=0,
\end{aligned}
$$

where we have used (2.21) in the last inequality. The above estimate implies that

$$
\lim _{a \nearrow a^{*}}\left(\varphi_{a}-\theta_{a}\right)=0,
$$

and Proposition 2.2 (i) is therefore proved in view of (2.25).

2. Note from (2.11) and (2.20) that $v_{a}(x)$ satisfies

$$
\begin{aligned}
& -\Delta v_{a}+i \alpha_{a}^{2} \Omega\left(x^{\perp} \cdot \nabla v_{a}\right)+\left[\frac{\alpha_{a}^{4} \Omega^{2}|x|^{2}}{4}+\alpha_{a}^{2} V_{\Omega}\left(\alpha_{a}\left(x+y_{0}\right)\right)\right] v_{a} \\
= & \alpha_{a}^{2} \mu_{a} v_{a}+a\left|v_{a}\right|^{2} v_{a} \text { in } \mathbb{R}^{2} .
\end{aligned}
$$

Similar to (2.35) below, by the comparison principle one can derive from (2.23) and (2.27) that as $a \nearrow a^{*}$,

$$
\left|v_{a}(x)\right| \leq C e^{-\frac{2}{3}|x|} \text { in } \mathbb{R}^{2} .
$$

It remains to prove the uniformly exponential decay of $\left|\nabla v_{a}\right|$ as $a \nearrow a^{*}$. Towards this aim, denoting $\partial_{j} v_{a}(x):=\frac{\partial v_{a}(x)}{\partial x_{j}}(j=1,2)$, we follow from (2.27) that for $j, l=1,2$,

$$
\begin{aligned}
& -\Delta \partial_{j} v_{a}+(-1)^{j+1} \alpha_{a}^{2} \Omega i \partial_{l} v_{a}+i \alpha_{a}^{2} \Omega\left(x^{\perp} \cdot \nabla \partial_{j} v_{a}\right) \\
& +\left(\frac{\alpha_{a}^{4} \Omega^{2}}{4}|x|^{2}+\alpha_{a}^{2} V_{\Omega}\left(\alpha_{a}\left(x+y_{0}\right)\right)-\alpha_{a}^{2} \mu_{a}-a\left|v_{a}\right|^{2}\right) \partial_{j} v_{a} \\
& +\left[\frac{\alpha_{a}^{4} \Omega^{2}}{2} x_{j}+\alpha_{a}^{2} \frac{\partial V_{\Omega}\left(\alpha_{a}\left(x+y_{0}\right)\right)}{\partial x_{j}}-2 a\left(\partial_{j} v_{a}, v_{a}\right)\right] v_{a}=0 \text { in } \mathbb{R}^{2}, \quad l \neq j,
\end{aligned}
$$

where $(f, g)=\operatorname{Re}(f \cdot \bar{g})$ denotes the real part of $f \cdot \bar{g}$. We then get that for $l \neq j$,

$$
\begin{aligned}
& -\frac{1}{2} \Delta\left|\partial_{j} v_{a}\right|^{2}+\left|\nabla \partial_{j} v_{a}\right|^{2}+(-1)^{j+1} \alpha_{a}^{2} \Omega\left(i \partial_{l} v_{a}, \partial_{j} v_{a}\right)-\alpha_{a}^{2} \Omega x^{\perp} \cdot\left(i \partial_{j} v_{a}, \nabla \partial_{j} v_{a}\right) \\
& +\left[\frac{\alpha_{a}^{4} \Omega^{2}}{4}|x|^{2}+\alpha_{a}^{2} V_{\Omega}\left(\alpha_{a}\left(x+y_{0}\right)\right)-\alpha_{a}^{2} \mu_{a}-a\left|v_{a}\right|^{2}\right]\left|\partial_{j} v_{a}\right|^{2} \\
& +\left(\frac{\alpha_{a}^{4} \Omega^{2}}{2} x_{j}+\alpha_{a}^{2} \frac{\partial V_{\Omega}\left(\alpha_{a}\left(x+y_{0}\right)\right)}{\partial x_{j}}\right)\left(v_{a}, \partial_{j} v_{a}\right)-2 a\left(\partial_{j} v_{a}, v_{a}\right)^{2}=0 \text { in } \mathbb{R}^{2}, \quad j, l=1,2 .
\end{aligned}
$$


By the inequality (2.4), we have

$$
\left|\nabla \partial_{j} v_{a}\right|^{2}-\alpha_{a}^{2} \Omega x^{\perp} \cdot\left(i \partial_{j} v_{a}, \nabla \partial_{j} v_{a}\right)+\frac{\alpha_{a}^{4} \Omega^{2}}{4}|x|^{2}\left|\partial_{j} v_{a}\right|^{2} \geq 0 \quad \text { in } \mathbb{R}^{2}, \quad j=1,2 .
$$

Under the assumption $(V)$, we also obtain from (2.28) that for $j=1,2$,

$$
\begin{aligned}
& \left|\left(\frac{\alpha_{a}^{4} \Omega^{2}}{2} x_{j}+\alpha_{a}^{2} \frac{\partial V_{\Omega}\left(\alpha_{a}\left(x+y_{0}\right)\right)}{\partial x_{j}}\right)\left(v_{a}, \partial_{j} v_{a}\right)\right| \\
\leq & \frac{\alpha_{a}^{4} \Omega^{2}}{2}\left(\frac{\left|x_{j}\right|^{2}\left|v_{a}\right|^{2}}{2}+\frac{\left|\partial_{j} v_{a}\right|^{2}}{2}\right)+\alpha_{a}^{2}\left(\frac{\left|\frac{\partial V_{\Omega}\left(\alpha_{a}\left(x+y_{0}\right)\right)}{\partial x_{j}}\right|^{2}\left|v_{a}\right|^{2}}{2}+\frac{\left|\partial_{j} v_{a}\right|^{2}}{2}\right) \\
\leq & C \alpha_{a}^{2} e^{-|x|}+C \alpha_{a}^{2}\left|\partial_{j} v_{a}\right|^{2} \text { in } \mathbb{R}^{2},
\end{aligned}
$$

and

$$
\begin{aligned}
& \left(-\alpha_{a}^{2} \mu_{a}-a\left|v_{a}\right|^{2}\right)\left(\left|\partial_{1} v_{a}\right|^{2}+\left|\partial_{2} v_{a}\right|^{2}\right)-2 a\left[\left(\partial_{1} v_{a}, v_{a}\right)^{2}+\left(\partial_{2} v_{a}, v_{a}\right)^{2}\right] \\
& +\alpha_{a}^{2} \Omega\left(i \partial_{2} v_{a}, \partial_{1} v_{a}\right)-\alpha_{a}^{2} \Omega\left(i \partial_{1} v_{a}, \partial_{2} v_{a}\right) \\
\geq & \left(\frac{3}{4}-3 a\left|v_{a}\right|^{2}\right)\left(\left|\partial_{1} v_{a}\right|^{2}+\left|\partial_{2} v_{a}\right|^{2}\right) \text { in } \mathbb{R}^{2},
\end{aligned}
$$

since $-\alpha_{a}^{2} \mu_{a} \rightarrow 1$ and $\alpha_{a}^{2} \rightarrow 0$ as $a \nearrow a^{*}$. Combining all above estimates, we get that as $a \nearrow a^{*}$,

$$
\left(-\frac{1}{2} \Delta+\frac{2}{3}-3 a\left|v_{a}\right|^{2}\right)\left|\nabla v_{a}\right|^{2} \leq C e^{-|x|} \text { in } \mathbb{R}^{2} .
$$

Because $v_{a}$ is bounded uniformly in $H^{1}\left(\mathbb{R}^{2}\right)$, one can deduce from De Giorgi-Nash-Moser theory [34, Theorem 4.1] that

$$
\max _{x \in B_{1}(\xi)}\left|\nabla v_{a}(x)\right|^{2} \leq C\left(\int_{B_{2}(\xi)}\left|\nabla v_{a}(x)\right|^{2} d x+\left\|e^{-|x|}\right\|_{L^{2}\left(B_{2}(\xi)\right)}\right) .
$$

Therefore, we derive from (2.23) and (2.32) that

$$
\left|\nabla v_{a}(x)\right|^{2} \leq C \text { and } \lim _{|x| \rightarrow \infty}\left|\nabla v_{a}(x)\right|^{2}=0 \text { as } a \nearrow a^{*} .
$$

Substituting (2.33) and (2.28) into (2.31) yields that as $a \nearrow a^{*}$,

$$
\left(-\Delta+\frac{4}{3}\right)\left|\nabla v_{a}(x)\right|^{2} \leq C e^{-|x|} \text { in } \mathbb{R}^{2} .
$$

Since $3 C e^{-|x|}>0$ is a supersolution of (2.34) in the sense that

$$
\left(-\Delta+\frac{4}{3}\right)\left(3 C e^{-|x|}\right) \geq C e^{-|x|} \text { in } \mathbb{R}^{2},
$$

we have

$$
\left(-\Delta+\frac{4}{3}\right)\left(\left|\nabla v_{a}(x)\right|^{2}-3 C e^{-|x|}\right) \leq 0 \text { in } \mathbb{R}^{2} \text { as } a \nearrow a^{*} .
$$

Note from (2.33) that $\lim _{|x| \rightarrow \infty}\left(\left|\nabla v_{a}(x)\right|^{2}-3 C e^{-|x|}\right)=0$ as $a \nearrow a^{*}$. We then deduce from the comparison principle that as $a \nearrow a^{*}$,

$$
\left|\nabla v_{a}(x)\right|^{2} \leq 3 C e^{-|x|} \text { in } \mathbb{R}^{2},
$$

and the gradient estimate of (2.24) hence holds.

Employing the non-degeneracy (2.7) of the linearized operator $\mathcal{L}$, we next establish the following refined $L^{\infty}$-uniform estimates of the imaginary part $I_{a}$ for $v_{a}$ : 
Lemma 2.3. Under the assumptions of Theorem 1.1, let $I_{a}$ be defined by (2.20). Then we have

$$
\left|I_{a}(x)\right| \leq C_{21}\left(\alpha_{a}\right) e^{-\frac{1}{4}|x|}, \quad\left|\nabla I_{a}(x)\right| \leq C_{22}\left(\alpha_{a}\right) e^{-\frac{1}{8}|x|} \quad \text { uniformly in } \mathbb{R}^{2} \text { as a } \nearrow a^{*} \text {, }
$$

where the constants $C_{21}\left(\alpha_{a}\right)>0$ and $C_{22}\left(\alpha_{a}\right)>0$ satisfy

$$
C_{21}\left(\alpha_{a}\right)=o\left(\alpha_{a}^{2}\right) \text { and } C_{22}\left(\alpha_{a}\right)=o\left(\alpha_{a}^{2}\right) \text { as a } \nearrow a^{*} .
$$

Proof. Denote the operator

$$
\mathcal{L}_{a}:=-\Delta+\frac{\alpha_{a}^{4} \Omega^{2}|x|^{2}}{4}+\alpha_{a}^{2} V_{\Omega}\left(\alpha_{a}\left(x+y_{0}\right)\right)-\alpha_{a}^{2} \mu_{a}-a\left|v_{a}\right|^{2} .
$$

Following (2.22) and (2.27), the imaginary part $I_{a}$ of $v_{a}$ satisfies

$$
\mathcal{L}_{a} I_{a}(x)=-\alpha_{a}^{2} \Omega\left(x^{\perp} \cdot \nabla R_{a}\right) \quad \text { in } \mathbb{R}^{2}, \quad \int_{\mathbb{R}^{2}} I_{a}(x) w(x) d x=0 .
$$

We first claim that as $a \nearrow a^{*}$,

$$
\left|\alpha_{a}^{2} \Omega\left(x^{\perp} \cdot \nabla R_{a}\right)\right| \leq C\left(\alpha_{a}\right) e^{-\frac{1}{4}|x|} \quad \text { uniformly in } \mathbb{R}^{2},
$$

where the constant $C\left(\alpha_{a}\right)>0$ satisfies $C\left(\alpha_{a}\right)=o\left(\alpha_{a}^{2}\right)$ as $a \nearrow a^{*}$. Actually, since $\left(x^{\perp} \cdot \nabla w\right) \equiv 0$ in $\mathbb{R}^{2}$, for any fixed large $R>0$, one can obtain from (2.3) and (2.24) that

$$
\left|x^{\perp} \cdot \nabla\left(R_{a}-\frac{w}{\sqrt{a^{*}}}\right)\right| \leq C e^{-\frac{R}{8}} e^{-\frac{1}{4}|x|} \text { in } \mathbb{R}^{2} / B_{R}(0) .
$$

On the other hand, we get from (2.24) that $v_{a}$ and $\left(x^{\perp} \cdot \nabla v_{a}\right)$ are bounded uniformly in $L^{\infty}\left(B_{R+1}(0)\right)$. Applying the $L^{p}$ estimate (cf. [26, Theorem 9.11]) to (2.27) yields that $v_{a}$ is bounded uniformly in $W^{2, q}\left(B_{R}(0)\right)$ for any $q>2$. Since the embedding $W^{2, q}\left(B_{R}(0)\right) \hookrightarrow$ $C^{1}\left(B_{R}(0)\right)$ is compact (cf. [26, Theorem 7.26]), there exist a subsequence $\left\{v_{a_{k}}\right\}$ of $\left\{v_{a}\right\}$ and $w_{0}(x) \in H^{1}\left(B_{R}(0)\right)$ such that

$$
v_{a_{k}}(x) \rightarrow w_{0}(x) \text { uniformly in } C^{1}\left(B_{R}(0)\right) \text { as } a_{k} \nearrow a^{*} .
$$

By the convergence (2.23) and the uniqueness of $w$, we conclude that $w_{0}(x) \equiv \frac{w(x)}{\sqrt{a^{*}}}$ in $B_{R}(0)$ and the above convergence hence holds for the whole sequence, i.e.,

$$
v_{a}(x) \rightarrow \frac{w}{\sqrt{a^{*}}} \text { uniformly in } C^{1}\left(B_{R}(0)\right) \text { as } a \nearrow a^{*} .
$$

Since $R>0$ is arbitrary, we deduce from (2.41) and (2.42) that the claim (2.40) holds true.

We next follow (2.40) to prove (2.36) and (2.37). Multiplying (2.39) by $I_{a}$ and integrating over $\mathbb{R}^{2}$, we get from (2.40) that

$$
\int_{\mathbb{R}^{2}}\left(\mathcal{L}_{a} I_{a}\right) I_{a} d x=-\alpha_{a}^{2} \Omega \int_{\mathbb{R}^{2}}\left(x^{\perp} \cdot \nabla R_{a}\right) I_{a} d x=o\left(\alpha_{a}^{2}\right)\left\|I_{a}\right\|_{L^{2}\left(\mathbb{R}^{2}\right)} \text { as } a \nearrow a^{*} .
$$

Following (2.7) and (2.23), since $\int_{\mathbb{R}^{2}} I_{a} w d x=0$, we also have

$$
\begin{aligned}
\int_{\mathbb{R}^{2}}\left(\mathcal{L}_{a} I_{a}\right) I_{a} d x & \geq \int_{\mathbb{R}^{2}}\left\{\left(\mathcal{L} I_{a}\right) I_{a}-\left(1+\alpha_{a}^{2} \mu_{a}\right) I_{a}^{2}-\left(a\left|v_{a}\right|^{2}-w^{2}\right) I_{a}^{2}\right\} d x \\
& =\int_{\mathbb{R}^{2}}\left(\mathcal{L} I_{a}\right) I_{a} d x+o(1) \int_{\mathbb{R}^{2}} I_{a}^{2} d x \geq \frac{\rho}{2}\left\|I_{a}\right\|_{H^{1}\left(\mathbb{R}^{2}\right)}^{2} \text { as } a \nearrow a^{*},
\end{aligned}
$$

where the constant $\rho>0$, independent of $0<a<a^{*}$, is given by (2.7). Hence, we obtain from (2.43) and (2.44) that

$$
\left\|I_{a}\right\|_{H^{1}\left(\mathbb{R}^{2}\right)}=o\left(\alpha_{a}^{2}\right) \text { as } a \nearrow a^{*} .
$$


On the other hand, we derive from (2.39) that $\left|I_{a}\right|^{2}$ satisfies

$$
\begin{aligned}
& {\left[-\frac{1}{2} \Delta+\left(\frac{\alpha_{a}^{4} \Omega^{2}}{4}|x|^{2}+\alpha_{a}^{2} V_{\Omega}\left(\alpha_{a}\left(x+y_{0}\right)\right)-\mu_{a} \alpha_{a}^{2}-a\left|v_{a}\right|^{2}\right)\right]\left|I_{a}\right|^{2}+\left|\nabla I_{a}\right|^{2} } \\
= & -\alpha_{a}^{2} \Omega\left(x^{\perp} \cdot \nabla R_{a}\right) I_{a} \text { in } \mathbb{R}^{2},
\end{aligned}
$$

which implies that

$$
-\frac{1}{2} \Delta\left|I_{a}\right|^{2}-\mu_{a} \alpha_{a}^{2}\left|I_{a}\right|^{2}-a\left|v_{a}\right|^{2}\left|I_{a}\right|^{2} \leq-\alpha_{a}^{2} \Omega\left(x^{\perp} \cdot \nabla R_{a}\right) I_{a} \text { in } \mathbb{R}^{2} .
$$

Following De Giorgi-Nash-Moser theory again (cf. [34, Theorem 4.1]), it follows from (2.46) that for any $\xi \in \mathbb{R}^{2}$,

$$
\sup _{x \in B_{1}(\xi)}\left|I_{a}(x)\right|^{2} \leq C\left(\left\|I_{a}\right\|_{L^{2}\left(B_{2}(\xi)\right)}^{2}+\left\|\alpha_{a}^{2} \Omega\left(x^{\perp} \cdot \nabla R_{a}\right) I_{a}\right\|_{L^{2}\left(B_{2}(\xi)\right)}\right) .
$$

Applying Proposition 2.2 we then deduce from (2.40), (2.45) and (2.47) that

$$
\left\|I_{a}\right\|_{L^{\infty}\left(\mathbb{R}^{2}\right)}=o\left(\alpha_{a}^{2}\right) \text { as } a \nearrow a^{*},
$$

and hence

$$
\left.|a| v_{a}\right|^{2} I_{a}-\alpha_{a}^{2} \Omega\left(x^{\perp} \cdot \nabla R_{a}\right) \mid \leq C_{0}\left(\alpha_{a}\right) e^{-\frac{1}{4}|x|} \text { uniformly in } \mathbb{R}^{2} \text { as } a \nearrow a^{*},
$$

where the constant $C_{0}\left(\alpha_{a}\right)>0$ satisfies $C_{0}\left(\alpha_{a}\right)=o\left(\alpha_{a}^{2}\right)$ as $a \nearrow a^{*}$. Applying the comparison principle to (2.46), we further get from (2.48) and (2.49) that

$$
\left|I_{a}(x)\right| \leq C_{1}\left(\alpha_{a}\right) e^{-\frac{1}{4}|x|} \quad \text { uniformly in } \mathbb{R}^{2} \text { as } a \nearrow a^{*},
$$

where the constant $C_{1}\left(\alpha_{a}\right)>0$ satisfies $C_{1}\left(\alpha_{a}\right)=o\left(\alpha_{a}^{2}\right)$ as $a \nearrow a^{*}$. Moreover, applying gradient estimates (see (3.15) in [26]) to the equation (2.39), we conclude from above that the gradient estimate of (2.36) and (2.37) holds true, which therefore completes the proof of Lemma 2.3.

\section{Analysis of the linearized problem (3.8)}

Following the $L^{\infty}$-uniform estimates of previous section, this section is concerned with the analysis of the linearized problem (3.8) defined below. In order to prove Theorem 1.1 by contradiction we first suppose that up to a constant phase, there exist two different minimizers $u_{1, a}$ and $u_{2, a}$ of $e_{F}(a)$ as $a \nearrow a^{*}$, in the sense that $u_{1, a} \not \equiv u_{2, a} e^{i \theta}$ for any constant phase $\theta=\theta(a) \in[0,2 \pi)$.

Recall that $\alpha_{a}>0$ is defined by (2.12), and $y_{0} \in \mathbb{R}^{2}$ denotes the unique non-degenerate critical point of $H(y)$. We then define for $j=1,2$,

$$
\tilde{u}_{j, a}(x):=\alpha_{a} u_{j, a}\left(\alpha_{a}\left(x+y_{0}\right)\right) e^{-i\left(\frac{\alpha_{a}^{2} \Omega}{2} x \cdot y_{0}^{\perp}\right)} e^{i \varphi_{j, a}}=R_{j, a}(x)+i I_{j, a}(x),
$$

where $R_{j, a}(x)$ and $I_{j, a}(x)$ denote the real and imaginary parts of $\tilde{u}_{j, a}(x)$, respectively, and the constant phase $\varphi_{j, a} \in[0,2 \pi)$ can be chosen properly such that

$$
\int_{\mathbb{R}^{2}} w(x) I_{j, a}(x) d x=0, \quad j=1,2 .
$$

We remark that (3.2) makes sense in view of (2.21) and the following fact: it yields from Proposition 2.2 that $\tilde{u}_{j, a}(x)$ satisfies for $j=1,2$,

$$
\lim _{a \nearrow a^{*}} \tilde{u}_{j, a}(x)=\frac{w(x)}{\sqrt{a^{*}}} \text { strongly in } L^{\infty}\left(\mathbb{R}^{2}, \mathbb{C}\right) \cap H^{1}\left(\mathbb{R}^{2}, \mathbb{C}\right) .
$$


Note from (2.11) and (3.1) that $\tilde{u}_{j, a}(x)$ satisfies the equation

$$
\begin{aligned}
& -\Delta \tilde{u}_{j, a}(x)+i \alpha_{a}^{2} \Omega\left(x^{\perp} \cdot \nabla \tilde{u}_{j, a}\right)+\left[\frac{\alpha_{a}^{4} \Omega^{2}|x|^{2}}{4}+\alpha_{a}^{2} V_{\Omega}\left(\alpha_{a}\left(x+y_{0}\right)\right)\right] \tilde{u}_{j, a}(x) \\
= & \alpha_{a}^{2} \mu_{j, a} \tilde{u}_{j, a}(x)+a\left|\tilde{u}_{j, a}\right|^{2} \tilde{u}_{j, a}(x) \quad \text { in } \mathbb{R}^{2}, \quad j=1,2 .
\end{aligned}
$$

Applying (3.2)-(3.4), we have the following estimates:

Lemma 3.1. Under the assumptions of Theorem 1.1, let $I_{j, a}(x)$ be defined by (3.1) for $j=1,2$. Then $I_{j, a}(x)$ satisfies for $j=1,2$,

$$
\left|I_{j, a}(x)\right| \leq C_{j 1}\left(\alpha_{a}\right) e^{-\frac{1}{4}|x|} \text { and }\left|\nabla I_{j, a}(x)\right| \leq C_{j 2}\left(\alpha_{a}\right) e^{-\frac{1}{8}|x|} \text { uniformly in } \mathbb{R}^{2} \text { as a } \nearrow a^{*} \text {, }
$$

where the constants $C_{j 1}\left(\alpha_{a}\right)>0$ and $C_{j 2}\left(\alpha_{a}\right)>0$ satisfy

$$
C_{j 1}\left(\alpha_{a}\right)=o\left(\alpha_{a}^{2}\right) \text { and } C_{j 2}\left(\alpha_{a}\right)=o\left(\alpha_{a}^{2}\right) \text { as a } \nearrow a^{*} .
$$

Since Lemma 3.1 can be established in the similar approach of proving Lemma 2.3, we omit the detailed proof for simplicity. We next define the following difference function:

$$
\eta_{a}(x):=\frac{\tilde{u}_{2, a}(x)-\tilde{u}_{1, a}(x)}{\left\|\tilde{u}_{2, a}(x)-\tilde{u}_{1, a}(x)\right\|_{L^{\infty}\left(\mathbb{R}^{2}\right)}}=\eta_{1, a}(x)+i \eta_{2, a}(x),
$$

where $\eta_{1, a}(x)$ and $\eta_{2, a}(x)$ denote the real and imaginary parts of $\eta_{a}(x)$, respectively. By the definition of $\eta_{a}(x)$, we deduce from (3.4) that $\eta_{a}(x)$ satisfies

$$
\begin{aligned}
& -\Delta \eta_{a}+i \alpha_{a}^{2} \Omega\left(x^{\perp} \cdot \nabla \eta_{a}\right)+\alpha_{a}^{2} V_{\Omega}\left(\alpha_{a}\left(x+y_{0}\right)\right) \eta_{a}+\frac{\alpha_{a}^{4} \Omega^{2}|x|^{2}}{4} \eta_{a} \\
= & \alpha_{a}^{2} \mu_{1, a} \eta_{a}-\frac{\tilde{u}_{2, a}}{2} \int_{\mathbb{R}^{2}} \tilde{f}_{a}\left(\left|\tilde{u}_{1, a}\right|^{2}+\left|\tilde{u}_{2, a}\right|^{2}\right) d x+\tilde{f}_{a} \tilde{u}_{1, a}+a\left|\tilde{u}_{2, a}\right|^{2} \eta_{a} \text { in } \mathbb{R}^{2},
\end{aligned}
$$

where $\tilde{f}_{a}$ is defined by

$$
\tilde{f}_{a}(x):=a \frac{\left|\tilde{u}_{2, a}(x)\right|^{2}-\left|\tilde{u}_{1, a}(x)\right|^{2}}{\left\|\tilde{u}_{2, a}-\tilde{u}_{1, a}\right\|_{L^{\infty}\left(\mathbb{R}^{2}\right)}}=a\left[\eta_{1, a}\left(R_{1, a}+R_{2, a}\right)+\eta_{2, a}\left(I_{1, a}+I_{2, a}\right)\right] .
$$

We next study the estimates of $\eta_{a}(x)$ as $a \nearrow a^{*}$.

Lemma 3.2. Suppose $\eta_{a}(x)$ is defined by (3.7), then there exists a constant $C>0$, independent of $0<a<a^{*}$, such that

$$
\left|\eta_{a}(x)\right| \leq C e^{-\frac{2}{3}|x|} \text { and }\left|\nabla \eta_{a}(x)\right| \leq C e^{-\frac{1}{2}|x|} \text { uniformly in } \mathbb{R}^{2} \text { as a } \nearrow a^{*} .
$$

Proof. We first address the estimate of $\eta_{a}(x)$ as $a \nearrow a^{*}$. Following (3.8), we have

$$
\begin{aligned}
& -\frac{1}{2} \Delta\left|\eta_{a}\right|^{2}+\left|\nabla \eta_{a}\right|^{2}-\alpha_{a}^{2} \Omega x^{\perp} \cdot\left(i \eta_{a}, \nabla \eta_{a}\right)+\left[\alpha_{a}^{2} V_{\Omega}\left(\alpha_{a}\left(x+y_{0}\right)\right)+\frac{\alpha_{a}^{4} \Omega^{2}|x|^{2}}{4}\right]\left|\eta_{a}\right|^{2} \\
= & \alpha_{a}^{2} \mu_{1, a}\left|\eta_{a}\right|^{2}-\frac{\left(\tilde{u}_{2, a}, \eta_{a}\right)}{2} \int_{\mathbb{R}^{2}} \tilde{f}_{a}\left(\left|\tilde{u}_{1, a}\right|^{2}+\left|\tilde{u}_{2, a}\right|^{2}\right) d x \\
& +\left(\tilde{f}_{a} \tilde{u}_{1, a}, \eta_{a}\right)+a\left|\tilde{u}_{2, a}\right|^{2}\left|\eta_{a}\right|^{2} \quad \text { in } \mathbb{R}^{2} .
\end{aligned}
$$

Using the diamagnetic inequality (2.4), we obtain that

$$
\left|\nabla \eta_{a}\right|^{2}-\alpha_{a}^{2} \Omega x^{\perp} \cdot\left(i \eta_{a}, \nabla \eta_{a}\right)+\frac{\alpha_{a}^{4} \Omega^{2}|x|^{2}}{4}\left|\eta_{a}\right|^{2} \geq 0 \quad \text { in } \mathbb{R}^{2}
$$

By the definition of $\tilde{f}_{a}$, we also get from Proposition 2.2 that there exists a constant $C>0$ such that

$$
\left\|\tilde{f}_{a}\right\|_{L^{\infty}\left(\mathbb{R}^{2}\right)} \leq C \quad \text { and } \quad\left|\int_{\mathbb{R}^{2}} \tilde{f}_{a}\left(\left|\tilde{u}_{1, a}\right|^{2}+\left|\tilde{u}_{2, a}\right|^{2}\right) d x\right| \leq C .
$$


Consequently, we obtain from Proposition 2.2 that

$$
\begin{aligned}
& \left|-\frac{\left(\tilde{u}_{2, a}, \eta_{a}\right)}{2} \int_{\mathbb{R}^{2}} \tilde{f}_{a}\left(\left|\tilde{u}_{1, a}\right|^{2}+\left|\tilde{u}_{2, a}\right|^{2}\right) d x+\left(\tilde{f}_{a} \tilde{u}_{1, a}, \eta_{a}\right)\right| \\
\leq & 2 \delta\left|\eta_{a}\right|^{2}+C(\delta)\left|\tilde{u}_{2, a}\right|^{2}+C(\delta)\left|\tilde{f}_{a}\right|^{2}\left|\tilde{u}_{1, a}\right|^{2} \\
\leq & 2 \delta\left|\eta_{a}\right|^{2}+C(\delta) e^{-\frac{4}{3}|x|} \quad \text { in } \mathbb{R}^{2},
\end{aligned}
$$

where $\delta>0$ is a small constant and $C(\delta)>0$ depends only on $\delta$. Recall that $-\alpha_{a}^{2} \mu_{1, a} \rightarrow 1$ as $a \nearrow a^{*}$ in view of Lemma 2.1 Setting $\delta=\frac{1}{16}$, we then deduce from (3.11)-(3.14) that as $a \nearrow a^{*}$,

$$
-\frac{1}{2} \Delta\left|\eta_{a}\right|^{2}+\frac{3}{4}\left|\eta_{a}\right|^{2} \leq C e^{-\frac{4}{3}|x|} \text { in } \mathbb{R}^{2}
$$

where Proposition 2.2 is used again. By the comparison principle, we conclude from above that there exists a constant $C>0$, independent of $0<a<a^{*}$, such that as $a \nearrow a^{*}$,

$$
\left|\eta_{a}(x)\right| \leq C e^{-\frac{2}{3}|x|} \quad \text { uniformly in } \mathbb{R}^{2},
$$

which implies that the estimate (3.10) of $\eta_{a}(x)$ holds true.

We next prove the estimate of $\left|\nabla \eta_{a}\right|$ as $a \nearrow a^{*}$. Actually, note from (3.8) and (3.13) that as a $\nearrow a^{*}$

$$
\begin{aligned}
\int_{\mathbb{R}^{2}}\left|\nabla \eta_{a}\right|^{2} d x \leq & \int_{\mathbb{R}^{2}}\left\{\left|\nabla \eta_{a}\right|^{2}+\left[\alpha_{a}^{2} V_{\Omega}\left(\alpha_{a}\left(x+y_{0}\right)\right)+\frac{\alpha_{a}^{4} \Omega^{2}|x|^{2}}{4}-\alpha_{a}^{2} \mu_{1, a}\right]\left|\eta_{a}\right|^{2}\right\} d x \\
= & \int_{\mathbb{R}^{2}}\left\{\alpha_{a}^{2} \Omega x^{\perp} \cdot\left(i \eta_{a}, \nabla \eta_{a}\right)+\left(\tilde{f}_{a} \tilde{u}_{1, a}, \eta_{a}\right)+a\left|\tilde{u}_{2, a}\right|^{2}\left|\eta_{a}\right|^{2}\right\} d x \\
& -\int_{\mathbb{R}^{2}} \frac{\left(\tilde{u}_{2, a}, \eta_{a}\right)}{2} d x \int_{\mathbb{R}^{2}} \tilde{f}_{a}\left(\left|\tilde{u}_{1, a}\right|^{2}+\left|\tilde{u}_{2, a}\right|^{2}\right) d x \\
\leq & \int_{\mathbb{R}^{2}}\left\{\frac{1}{2}\left|\nabla \eta_{a}\right|^{2}+\frac{\alpha_{a}^{4} \Omega^{2}|x|^{2}}{2}\left|\eta_{a}\right|^{2}\right\} d x+C \\
\leq & \frac{1}{2} \int_{\mathbb{R}^{2}}\left|\nabla \eta_{a}\right|^{2} d x+C
\end{aligned}
$$

where the estimate (3.15) is also used. Following the above estimate, there exists a constant $C>0$ such that as $a \nearrow a^{*}$,

$$
\left\|\nabla \eta_{a}(x)\right\|_{L^{2}\left(\mathbb{R}^{2}\right)} \leq C .
$$

For convenience, we now denote

$$
\begin{gathered}
\partial_{j} \eta_{a}:=\frac{\partial \eta_{a}(x)}{\partial x_{j}}, V_{a}(x):=\alpha_{a}^{2} V_{\Omega}\left(\alpha_{a}\left(x+y_{0}\right)\right)+\frac{\alpha_{a}^{4} \Omega^{2}|x|^{2}}{4}, \\
F_{a}(x):=-\frac{\tilde{u}_{2, a}}{2} \int_{\mathbb{R}^{2}} \tilde{f}_{a}\left(\left|\tilde{u}_{1, a}\right|^{2}+\left|\tilde{u}_{2, a}\right|^{2}\right) d x+\tilde{f}_{a} \tilde{u}_{1, a} .
\end{gathered}
$$

It follows from (3.8) that for $j, l=1,2$,

$$
\begin{aligned}
& -\Delta \partial_{j} \eta_{a}+i \alpha_{a}^{2} \Omega\left(x^{\perp} \cdot \nabla \partial_{j} \eta_{a}\right)+(-1)^{j+1} i \partial_{l} \eta_{a}+V_{a}(x) \partial_{j} \eta_{a}+\frac{\partial V_{a}}{\partial x_{j}} \eta_{a} \\
= & \alpha_{a}^{2} \mu_{1, a} \partial_{j} \eta_{a}+\frac{\partial F_{a}}{\partial x_{j}}+a\left|\tilde{u}_{2, a}\right|^{2} \partial_{j} \eta_{a}+a \frac{\partial\left|\tilde{u}_{2, a}\right|^{2}}{\partial x_{j}} \eta_{a} \text { in } \mathbb{R}^{2}, j \neq l .
\end{aligned}
$$

This further implies that for $j, l=1,2$,

$$
\begin{aligned}
& -\frac{1}{2} \Delta\left|\partial_{j} \eta_{a}\right|^{2}+\left|\nabla \partial_{j} \eta_{a}\right|^{2}-\alpha_{a}^{2} \Omega x^{\perp} \cdot\left(i \partial_{j} \eta_{a}, \nabla \partial_{j} \eta_{a}\right) \\
& +(-1)^{j+1}\left(i \partial_{l} \eta_{a}, \partial_{j} \eta_{a}\right)+V_{a}(x)\left|\partial_{j} \eta_{a}\right|^{2}+\frac{\partial V_{a}}{\partial x_{j}}\left(\eta_{a}, \partial_{j} \eta_{a}\right) \\
= & \alpha_{a}^{2} \mu_{1, a}\left|\partial_{j} \eta_{a}\right|^{2}+\left(\frac{\partial F_{a}}{\partial x_{j}}, \partial_{j} \eta_{a}\right)+a\left|\tilde{u}_{2, a}\right|^{2}\left|\partial_{j} \eta_{a}\right|^{2}+a \frac{\partial\left|\tilde{u}_{2, a}\right|^{2}}{\partial x_{j}}\left(\eta_{a}, \partial_{j} \eta_{a}\right) \text { in } \mathbb{R}^{2}, j \neq l .
\end{aligned}
$$


Applying the comparison principle, we follow (3.15)-(3.17) to finish the proof as follows:

By Cauchy's inequality, we get that

$$
\alpha_{a}^{2} \Omega x^{\perp} \cdot\left(i \partial_{j} \eta_{a}, \nabla \partial_{j} \eta_{a}\right) \leq \frac{\alpha_{a}^{4} \Omega^{2}|x|^{2}}{4}\left|\partial_{j} \eta_{a}\right|^{2}+\left|\nabla \partial_{j} \eta_{a}\right|^{2} \quad \text { in } \mathbb{R}^{2} .
$$

Under the assumption $(V)$, we obtain from (2.24) and (3.15) that

$$
\begin{aligned}
\frac{\partial V_{a}}{\partial x_{j}}\left(\eta_{a}, \partial_{j} \eta_{a}\right) & \leq \delta\left|\partial_{j} \eta_{a}\right|^{2}+C(\delta)\left|\frac{\partial V_{a}}{\partial x_{j}}\right|^{2}\left|\eta_{a}\right|^{2} \\
& \leq \delta\left|\partial_{j} \eta_{a}\right|^{2}+C(\delta) e^{-|x|} \text { in } \mathbb{R}^{2},
\end{aligned}
$$

and

$$
\begin{aligned}
a \frac{\partial\left|u_{2, a}\right|^{2}}{\partial x_{j}}\left(\eta_{a}, \partial_{j} \eta_{a}\right) & \leq \delta\left|\partial_{j} \eta_{a}\right|^{2}+C(\delta) a^{2}\left|\frac{\partial\left|\tilde{u}_{2, a}\right|^{2}}{\partial x_{j}}\right|^{2}\left|\eta_{a}\right|^{2} \\
& \leq \delta\left|\partial_{j} \eta_{a}\right|^{2}+C(\delta) e^{-|x|} \text { in } \mathbb{R}^{2},
\end{aligned}
$$

where $\delta>0$ is a small constant and $C(\delta)>0$ depends only on $\delta$. Similarly, one can derive from (3.9) and (3.13) that

$$
\begin{aligned}
\left(\frac{\partial F_{a}}{\partial x_{j}}, \partial_{j} \eta_{a}\right) & \leq 2 \delta\left|\partial_{j} \eta_{a}\right|^{2}+C(\delta)\left(\left|\frac{\partial \tilde{u}_{2, a}}{\partial x_{j}}\right|^{2}+\left|\frac{\partial \tilde{u}_{1, a}}{\partial x_{j}}\right|^{2}\right)+\left(\frac{\partial \tilde{f}_{a}}{\partial x_{j}} \tilde{u}_{1, a}, \partial_{j} \eta_{a}\right) \\
& \leq 3 \delta\left|\partial_{j} \eta_{a}\right|^{2}+C(\delta) e^{-|x|}+C e^{-|x|}\left|\partial_{j} \eta_{a}\right|^{2} \quad \text { in } \mathbb{R}^{2},
\end{aligned}
$$

where the estimates (2.24) and (3.16) are also used. Moreover, since $-\alpha_{a}^{2} \mu_{1, a} \rightarrow 1$ as $a \nearrow a^{*}$, we get that as $a \nearrow a^{*}$,

$$
\begin{aligned}
& -\alpha_{a}^{2} \mu_{1, a}\left(\left|\partial_{1} \eta_{a}\right|^{2}+\left|\partial_{2} \eta_{a}\right|^{2}\right)+\alpha_{a}^{2}\left(i \partial_{2} \eta_{a}, \partial_{1} \eta_{a}\right)-\alpha_{a}^{2}\left(i \partial_{1} \eta_{a}, \partial_{2} \eta_{a}\right) \\
\geq & \left(-\alpha_{a}^{2} \mu_{1, a}-\alpha_{a}^{2}\right)\left(\left|\partial_{1} \eta_{a}\right|^{2}+\left|\partial_{2} \eta_{a}\right|^{2}\right) \geq \frac{3}{4}\left(\left|\partial_{1} \eta_{a}\right|^{2}+\left|\partial_{2} \eta_{a}\right|^{2}\right) .
\end{aligned}
$$

Following (3.18)-(3.22) with $\delta=\frac{1}{60}$, we conclude from (3.17) that as $a \nearrow a^{*}$,

$$
-\frac{1}{2} \Delta\left|\nabla \eta_{a}\right|^{2}+\frac{2}{3}\left|\nabla \eta_{a}\right|^{2}-C e^{-|x|}\left|\nabla \eta_{a}\right|^{2} \leq C e^{-|x|} \text { in } \mathbb{R}^{2} .
$$

Applying De Giorgi-Nash-Moser theory again, we then deduce from (3.23) that for any $\xi \in \mathbb{R}^{2}$,

$$
\max _{x \in B_{1}(\xi)}\left|\nabla \eta_{a}(x)\right|^{2} \leq C\left(\int_{B_{2}(\xi)}\left|\nabla \eta_{a}(x)\right|^{2} d x+\left\|e^{-|x|}\right\|_{L^{2}\left(B_{2}(\xi)\right)}\right) \text { as a } \nearrow a^{*} .
$$

Applying (3.16), we thus obtain from above that as $a \nearrow a^{*}$,

$$
\left\|\nabla \eta_{a}(x)\right\|_{L^{\infty}\left(\mathbb{R}^{2}\right)} \leq C \text { and } \lim _{|x| \rightarrow \infty}\left|\nabla \eta_{a}(x)\right|=0 .
$$

Following (3.24), we derive from (3.23) that as $a \nearrow a^{*}$,

$$
-\frac{1}{2} \Delta\left|\nabla \eta_{a}\right|^{2}+\frac{2}{3}\left|\nabla \eta_{a}\right|^{2} \leq C e^{-|x|}\left|\nabla \eta_{a}\right|^{2}+C e^{-|x|} \leq C e^{-|x|} \text { in } \mathbb{R}^{2},
$$

from which the desired estimate (3.10) of $\left|\nabla \eta_{a}\right|$ can be proved by the comparison principle. This completes the proof of Lemma 3.2

Lemma 3.3. Suppose $\eta_{a}(x)$ is defined by 3.7). Then there exist a subsequence of $\left\{\eta_{a}\right\}$ (still denoted by $\left.\left\{\eta_{a}\right\}\right)$ and some constants $b_{0}, b_{1}$ and $b_{2}$ such that

$$
\eta_{a}(x)=b_{0}(w+x \cdot \nabla w)+\sum_{j=1}^{2} b_{j} \frac{\partial w}{\partial x_{j}}+M_{a}(x) \text { uniformly in } \mathbb{R}^{2} \text { as a } \nearrow a^{*},
$$


where the lower order term $M_{a}(x)$ satisfies

$$
\left|M_{a}(x)\right| \leq C_{M}\left(\alpha_{a}\right) e^{-\frac{1}{2}|x|}, \quad\left|\nabla M_{a}(x)\right| \leq C_{M}\left(\alpha_{a}\right) e^{-\frac{1}{4}|x|} \text { uniformly in } \mathbb{R}^{2} \text { as a } \nearrow a^{*}
$$

for some constant $C_{M}\left(\alpha_{a}\right)>0$ satisfying $C_{M}\left(\alpha_{a}\right)=o(1)$ as a $\nearrow a^{*}$.

Proof. We first claim that there exist a subsequence of $\left\{\eta_{a}\right\}$ (still denoted by $\left\{\eta_{a}\right\}$ ) and $b_{0}, b_{1}$ and $b_{2}$ such that

$$
\eta_{a}(x) \rightarrow b_{0}(w+x \cdot \nabla w)+\sum_{j=1}^{2} b_{j} \frac{\partial w}{\partial x_{j}} \quad \text { uniformly in } C_{l o c}^{1}\left(\mathbb{R}^{2}, \mathbb{C}\right) \text { as } a \nearrow a^{*} .
$$

Indeed, we note from Lemma 3.2 that the term $\left(x^{\perp} \cdot \nabla \eta_{a}\right)$ is bounded uniformly and decays exponentially for sufficiently large $|x|$ as $a \nearrow a^{*}$. By the standard elliptic regularity (cf. [26]), it then follows from (3.8) and (3.13) that $\eta_{a} \in C_{\text {loc }}^{1, \alpha}\left(\mathbb{R}^{2}, \mathbb{C}\right)$ and $\left\|\eta_{a}\right\|_{C_{l o c}^{1, \alpha}\left(\mathbb{R}^{2}, \mathbb{C}\right)} \leq C$ uniformly as $a \nearrow a^{*}$ for some $\alpha \in(0,1)$. By the equation (3.8), this implies that there exists a subsequence of $\left\{\eta_{a}\right\}$ (still denoted by $\left\{\eta_{a}\right\}$ ) such that $\eta_{a}=\eta_{1, a}+i \eta_{2, a} \rightarrow \eta_{0}=\eta_{1}+i \eta_{2} \in H^{1}\left(\mathbb{R}^{2}, \mathbb{C}\right.$ ) uniformly in $C_{l o c}^{1}\left(\mathbb{R}^{2}, \mathbb{C}\right)$ as $a \nearrow a^{*}$, where $\eta_{0}$ is a weak solution of

$$
-\Delta \eta_{0}+\eta_{0}-2 w^{2} \eta_{1}-w^{2} \eta_{0}=-\left(\frac{2}{a^{*}} \int_{\mathbb{R}^{2}} w^{3} \eta_{1} d x\right) w \text { in } \mathbb{R}^{2},
$$

which further implies that $\left(\eta_{1}, \eta_{2}\right)$ satisfies the following system

$$
\left\{\begin{array}{l}
-\Delta \eta_{1}+\eta_{1}-3 w^{2} \eta_{1}=-\left(\frac{2}{a^{*}} \int_{\mathbb{R}^{2}} w^{3} \eta_{1} d x\right) w \text { in } \mathbb{R}^{2} \\
-\Delta \eta_{2}+\eta_{2}-w^{2} \eta_{2}=0 \text { in } \mathbb{R}^{2} .
\end{array}\right.
$$

Notice from (3.2) and (3.7) that $\int_{\mathbb{R}^{2}} w(x) \eta_{2, a}(x) d x \equiv 0$, which further gives that $\int_{\mathbb{R}^{2}} w(x) \eta_{2}(x) d x=$ 0 , in view of the fact that $\eta_{2, a} \rightarrow \eta_{2}$ in $C_{l o c}^{1}\left(\mathbb{R}^{2}\right)$ as $a \nearrow a^{*}$. Since

$$
\left(-\Delta+1-3 w^{2}\right)(w+x \cdot \nabla w)=-2 w,
$$

we conclude from (2.6), (2.9) and (3.28) that there exist some constants $b_{0}, b_{1}$ and $b_{2}$ such that

$$
\eta_{1}=b_{0}(w+x \cdot \nabla w)+\sum_{i=1}^{2} b_{i} \frac{\partial w}{\partial x_{i}} \quad \text { and } \quad \eta_{2} \equiv 0 \quad \text { in } \mathbb{R}^{2},
$$

and the claim (3.27) is therefore proved.

On the other hand, for any fixed sufficiently large $R>0$, we derive from (2.3) and (3.10) that as $a \nearrow a^{*}$,

$$
\left|\eta_{a}(x)-\left[b_{0}(w+x \cdot \nabla w)+\sum_{j=1}^{2} b_{j} \frac{\partial w}{\partial x_{j}}\right]\right| \leq C e^{-\frac{1}{12} R} e^{-\frac{1}{2}|x|} \quad \text { in } \mathbb{R}^{2} / B_{R}(0),
$$

and

$$
\left|\nabla \eta_{a}(x)-\nabla\left[b_{0}(w+x \cdot \nabla w)+\sum_{j=1}^{2} b_{j} \frac{\partial w}{\partial x_{j}}\right]\right| \leq C e^{-\frac{1}{4} R} e^{-\frac{1}{4}|x|} \quad \text { in } \mathbb{R}^{2} / B_{R}(0) .
$$

Since $R>0$ is arbitrary, together with (3.27), we conclude from (3.29) and (3.30) that (3.26) holds true, which completes the proof of Lemma 3.3 .

Based on Lemma 3.3, we next establish the following refined estimate of $\eta_{a}$ as $a \nearrow a^{*}$. 
Proposition 3.4. Suppose $\left\{\eta_{a}\right\}$ is the subsequence obtained in Lemma 3.3. Then the imaginary part $\eta_{2, a}$ of $\eta_{a}$ satisfies

$$
\eta_{2, a}(x)=\frac{\alpha_{a}^{2} \Omega}{2}\left(-b_{1} x_{2}+b_{2} x_{1}\right) w(x)+E_{a}(x) \text { uniformly in } \mathbb{R}^{2} \text { as a } \nearrow a^{*},
$$

where $\left(x_{1}, x_{2}\right)=x \in \mathbb{R}^{2}$, the constants $b_{1}$ and $b_{2}$ are as in Lemma 3.3. and the lower order term $E_{a}(x)$ satisfies

$$
\left|E_{a}(x)\right| \leq C_{E}\left(\alpha_{a}\right) e^{-\frac{1}{8}|x|}, \quad\left|\nabla E_{a}(x)\right| \leq C_{E}\left(\alpha_{a}\right) e^{-\frac{1}{16}|x|} \text { uniformly in } \mathbb{R}^{2} \text { as a } \nearrow a^{*}
$$

for some constant $C_{E}\left(\alpha_{a}\right)>0$ satisfying $C_{E}\left(\alpha_{a}\right)=o\left(\alpha_{a}^{2}\right)$ as a $\nearrow a^{*}$.

Proof. We first get from (3.8) that

$$
\mathcal{L}_{2, a} \eta_{2, a}=-\alpha_{a}^{2} \Omega\left(x^{\perp} \cdot \nabla \eta_{1, a}\right)-\frac{I_{2, a}}{2} \int_{\mathbb{R}^{2}} \tilde{f}_{a}\left(\left|\tilde{u}_{1, a}\right|^{2}+\left|\tilde{u}_{2, a}\right|^{2}\right) d x+\tilde{f}_{a} I_{1, a} \text { in } \mathbb{R}^{2},
$$

where $\tilde{f}_{a}$ is defined by (3.9) and $\mathcal{L}_{j, a}$ is defined for $j=1,2$,

$$
\mathcal{L}_{j, a}:=-\Delta+\alpha_{a}^{2} V_{\Omega}\left(\alpha_{a}\left(x+y_{0}\right)\right)+\frac{\alpha_{a}^{4} \Omega^{2}|x|^{2}}{4}-\alpha_{a}^{2} \mu_{j, a}-a\left|\tilde{u}_{j, a}\right|^{2} .
$$

Set

$$
G_{a}(x):=-\frac{I_{2, a}}{2} \int_{\mathbb{R}^{2}} \tilde{f}_{a}\left(\left|\tilde{u}_{1, a}\right|^{2}+\left|\tilde{u}_{2, a}\right|^{2}\right) d x+\tilde{f}_{a} I_{1, a} .
$$

We then get from (3.25) and (3.33) that $\eta_{2, a}$ satisfies

$$
\mathcal{L}_{2, a} \eta_{2, a}=-\alpha_{a}^{2} \Omega\left[-b_{1} \frac{\partial w}{\partial x_{2}}+b_{2} \frac{\partial w}{\partial x_{1}}+\operatorname{Re}\left(x^{\perp} \cdot \nabla M_{a}\right)\right]+G_{a}(x) \text { in } \mathbb{R}^{2}, \int_{\mathbb{R}^{2}} \eta_{2, a} w d x=0,
$$

where (3.2) is used and $\operatorname{Re}(\cdot)$ denotes the real part. Here the constants $b_{1}$ and $b_{2}$ are as in Lemma 3.3. Note that $-\frac{1}{2} x_{j} w(x)$ is the unique solution of the following equation:

$$
-\Delta u+u-w^{2} u=\frac{\partial w}{\partial x_{j}}, \quad \int_{\mathbb{R}^{2}} u w d x=0, \quad j=1,2 .
$$

Denote

$$
E_{a}(x):=\eta_{2, a}(x)-\frac{\alpha_{a}^{2} \Omega}{2}\left(-b_{1} x_{2}+b_{2} x_{1}\right) w(x) .
$$

Applying (3.36), we thus obtain from (3.35) that $E_{a}(x)$ satisfies

$$
\mathcal{L}_{2, a} E_{a}(x)=\frac{\alpha_{a}^{2} \Omega}{2}\left(\mathcal{L}-\mathcal{L}_{2, a}\right)\left[\left(-b_{1} x_{2}+b_{2} x_{1}\right) w\right]-\alpha_{a}^{2} \Omega \operatorname{Re}\left(x^{\perp} \cdot \nabla M_{a}\right)+G_{a}(x) \text { in } \mathbb{R}^{2},
$$

and

$$
\int_{\mathbb{R}^{2}} E_{a}(x) w(x) d x=0,
$$

where the operator $\mathcal{L}$ is defined by (2.5).

We now estimate the right hand side of (3.37). By the definition of $\mathcal{L}_{2, a}$ in (3.34), we have

$$
\frac{\alpha_{a}^{2} \Omega}{2}\left|\left(\mathcal{L}_{2, a}-\mathcal{L}\right)\left[\left(-b_{1} x_{2}+b_{2} x_{1}\right) w\right]\right| \leq C\left(\alpha_{a}\right) e^{-\frac{1}{4}|x|} \text { uniformly in } \mathbb{R}^{2} \text { as } a \nearrow a^{*} .
$$

where $C\left(\alpha_{a}\right)>0$ satisfies

$$
C\left(\alpha_{a}\right)=o\left(\alpha_{a}^{2}\right) \text { as } a \nearrow a^{*} .
$$

Applying Lemma 3.3 for the estimate of $\nabla M_{a}$, we also obtain that

$$
\left|\operatorname{Re}\left(\alpha_{a}^{2} \Omega x^{\perp} \cdot \nabla M_{a}\right)\right| \leq C\left(\alpha_{a}\right) e^{-\frac{1}{8}|x|} \text { uniformly in } \mathbb{R}^{2} \text { as } a \nearrow a^{*},
$$


where $C\left(\alpha_{a}\right)>0$ also satisfies (3.39). Moreover, it follows from Lemma 3.1 and (3.13) that

$$
\left|G_{a}(x)\right| \leq C\left(\alpha_{a}\right) e^{-\frac{1}{4}|x|} \text { uniformly in } \mathbb{R}^{2} \text { as } a \nearrow a^{*},
$$

where $C\left(\alpha_{a}\right)>0$ satisfies (3.39) again. Following above estimates, we deduce from (3.37) that

$$
\begin{aligned}
\left|\mathcal{L}_{2, a} E_{a}(x)\right| & =\left|\frac{\alpha_{a}^{2} \Omega}{2}\left(\mathcal{L}_{2, a}-\mathcal{L}\right)\left[\left(-b_{1} x_{2}+b_{2} x_{1}\right) w\right]-\operatorname{Re}\left(\alpha_{a}^{2} \Omega x^{\perp} \cdot \nabla M_{a}\right)+G_{a}(x)\right| \\
& \leq C\left(\alpha_{a}\right) e^{-\frac{1}{8}|x|} \text { uniformly in } \mathbb{R}^{2} \text { as } a \nearrow a^{*},
\end{aligned}
$$

where $C\left(\alpha_{a}\right)>0$ satisfies (3.39). By the same argument of Lemma 2.3. Proposition 3.4 is therefore complete in view of (3.37), (3.38) and (3.40).

\section{Proof of Theorem 1.1}

As outlined in the introduction, in this section we are ready to complete the proof of Theorem 1.1 on the local uniqueness of minimizers for $e_{F}(a)$ as $a \nearrow a^{*}$.

Proof of Theorem 1.1. By contradiction, suppose that, up to a constant phase, there exist two different minimizers $u_{1, a}$ and $u_{2, a}$ of $e_{F}(a)$ as $a \nearrow a^{*}$. This means that $u_{1, a} \not \equiv u_{2, a} e^{i \theta}$ for any constant phase $\theta=\theta(a) \in[0,2 \pi)$. Recall that $\tilde{u}_{j, a}:=R_{j, a}(x)+i I_{j, a}(x)$ defined by (3.1) satisfies the following equation

$$
\begin{gathered}
-\Delta \tilde{u}_{j, a}(x)+i \alpha_{a}^{2} \Omega\left(x^{\perp} \cdot \nabla \tilde{u}_{j, a}\right)+\left[\frac{\alpha_{a}^{4} \Omega^{2}|x|^{2}}{4}+\alpha_{a}^{2} V_{\Omega}\left(\alpha_{a}\left(x+y_{0}\right)\right)\right] \tilde{u}_{j, a}(x) \\
=\alpha_{a}^{2} \mu_{j, a} \tilde{u}_{j, a}(x)+a\left|\tilde{u}_{j, a}\right|^{2} \tilde{u}_{j, a}(x) \quad \text { in } \mathbb{R}^{2}, \quad j=1,2,
\end{gathered}
$$

where $\mu_{j, a} \in \mathbb{R}$ satisfies

$$
\mu_{j, a}=e_{F}(a)-\frac{a}{2 \alpha_{a}^{2}} \int_{\mathbb{R}^{2}}\left|\tilde{u}_{j, a}\right|^{4} d x .
$$

Thus, the real part $R_{j, a}(x)$ of $\tilde{u}_{j, a}(x)$ satisfies

$$
\begin{gathered}
-\Delta R_{j, a}(x)-\alpha_{a}^{2} \Omega\left(x^{\perp} \cdot \nabla I_{j, a}\right)+\left[\frac{\alpha_{a}^{4} \Omega^{2}|x|^{2}}{4}+\alpha_{a}^{2} V_{\Omega}\left(\alpha_{a}\left(x+y_{0}\right)\right)\right] R_{j, a}(x) \\
=\alpha_{a}^{2} \mu_{j, a} R_{j, a}(x)+a\left|\tilde{u}_{j, a}\right|^{2} R_{j, a}(x) \quad \text { in } \mathbb{R}^{2}, \quad j=1,2 .
\end{gathered}
$$

Applying (4.2), one can derive from Lemmas 3.1 and 3.3 that as $a \nearrow a^{*}$,

$$
\begin{aligned}
& \frac{\alpha_{a}^{2}\left(\mu_{2, a}-\mu_{1, a}\right)}{\left\|\tilde{u}_{2, a}-\tilde{u}_{1, a}\right\|_{L^{\infty}\left(\mathbb{R}^{2}\right)}} \\
= & -\frac{a}{2} \int_{\mathbb{R}^{2}}\left[\eta_{1, a}\left(R_{1, a}+R_{2, a}\right)+\eta_{2, a}\left(I_{1, a}+I_{2, a}\right)\right] \cdot\left(\left|\tilde{u}_{2, a}\right|^{2}+\left|\tilde{u}_{1, a}\right|^{2}\right) d x \\
= & -\frac{2 a}{\left(a^{*}\right)^{3 / 2}} \int_{\mathbb{R}^{2}}\left[b_{0}(w+x \cdot \nabla w)+\sum_{j=1}^{2} b_{j} \frac{\partial w}{\partial x_{j}}\right] w^{3} d x+o(1)=b_{0} O(1)+o(1),
\end{aligned}
$$

where the constants $b_{0}, b_{1}$ and $b_{2}$ are as in (3.25).

We next prove $b_{i}=0$ for (4.4), where $i=0,1,2$. Our main idea is to derive the linear equations of $b_{i}$ through Pohozaev identities of $R_{j, a}$ satisfying (4.3), where $i=0,1,2$ and $j=1,2$. More precisely, we shall first construct Pohozaev identities of $R_{j, a}$, which are involved with the homogeneous potential $h(x)$ and the terms produced by the rotation. Applying Lemma 3.1 and Proposition 3.4 we shall prove that the terms produced by the rotation are lower order as $a \nearrow a^{*}$, which then yield the quantitative relationships between $\eta_{1}$ satisfying (3.28) and the homogeneous potential $h(x)$. Inserting (3.25) into those quantitative relationships, we further obtain the linear 
equations of $b_{i}$, from which we shall finally derive that $b_{i}=0$ for $i=0,1,2$ under some suitable assumptions on $h(x)$. We now carry out the above idea by the following three steps:

Step 1. We claim that the constants $b_{0}, b_{1}$ and $b_{2}$ defined in (3.25) satisfy the following equations:

$$
b_{0} \int_{\mathbb{R}^{2}} \frac{\partial h\left(x+y_{0}\right)}{\partial x_{l}}\left(x \cdot \nabla w^{2}\right) d x+\sum_{j=1}^{2} b_{j} \int_{\mathbb{R}^{2}} \frac{\partial h\left(x+y_{0}\right)}{\partial x_{l}} \frac{\partial w^{2}}{\partial x_{j}} d x=0, \quad l=1,2 .
$$

The intuition behind (4.5) is as follows: one can obtain the relationship between the homogeneous potential $h(x)$ and the coefficients $b_{0}, b_{1}$ and $b_{2}$ by constructing Pohozaev identities; Moreover, if one can further obtain that $b_{0}=0$ (see Step 2 below), then it implies from (4.5) that $b_{1}=b_{2}=0$ under the non-degeneracy assumption of the critical point $y_{0}$ for $H(y)$.

To prove the above claim (4.5), we first multiply the equation (4.3) by $\frac{\partial R_{j, a}(x)}{\partial x_{l}}$ to get that

$$
\begin{gathered}
-\Delta R_{j, a}(x) \frac{\partial R_{j, a}(x)}{\partial x_{l}}-\alpha_{a}^{2} \Omega\left(x^{\perp} \cdot \nabla I_{j, a}\right) \frac{\partial R_{j, a}(x)}{\partial x_{l}} \\
+\frac{1}{2}\left[\frac{\alpha_{a}^{4} \Omega^{2}|x|^{2}}{4}+\alpha_{a}^{2} V_{\Omega}\left(\alpha_{a}\left(x+y_{0}\right)\right)\right] \frac{\partial\left|R_{j, a}\right|^{2}}{\partial x_{l}} \\
=\frac{1}{2}\left(\alpha_{a}^{2} \mu_{j, a}+a\left|\tilde{u}_{j, a}\right|^{2}\right) \frac{\partial\left|R_{j, a}\right|^{2}}{\partial x_{l}} \text { in } \mathbb{R}^{2}, j, l=1,2 .
\end{gathered}
$$

Following the exponential decay (2.24), we calculate that for $j=1,2$,

$$
\begin{aligned}
-\int_{\mathbb{R}^{2}} \Delta R_{j, a} \frac{\partial R_{j, a}(x)}{\partial x_{l}} d x & =-\lim _{R \rightarrow \infty} \int_{B_{R}(0)} \Delta R_{j, a} \frac{\partial R_{j, a}(x)}{\partial x_{l}} d x \\
& =-\lim _{R \rightarrow \infty} \int_{\partial B_{R}(0)}\left(\frac{\partial R_{j, a}(x)}{\partial \nu} \frac{\partial R_{j, a}(x)}{\partial x_{l}}-\frac{1}{2}\left|\nabla R_{j, a}\right|^{2} \nu_{l}\right) d S=0,
\end{aligned}
$$

where $\nu=\left(\nu_{1}, \nu_{2}\right)$ denotes the outward unit of $\partial B_{R}(0)$. Similarly, we have

$$
\int_{\mathbb{R}^{2}}\left|\tilde{u}_{j, a}\right|^{2} \frac{\partial\left|R_{j, a}\right|^{2}}{\partial x_{l}} d x=\int_{\mathbb{R}^{2}}\left(R_{j, a}^{2}+I_{j, a}^{2}\right) \frac{\partial\left|R_{j, a}\right|^{2}}{\partial x_{l}} d x=\int_{\mathbb{R}^{2}} I_{j, a}^{2} \frac{\partial\left|R_{j, a}\right|^{2}}{\partial x_{l}} d x
$$

and

$$
\frac{1}{2} \int_{\mathbb{R}^{2}}\left[\frac{\alpha_{a}^{4} \Omega^{2}|x|^{2}}{4}+\alpha_{a}^{2} V_{\Omega}\left(\alpha_{a}\left(x+y_{0}\right)\right)\right] \frac{\partial\left|R_{j, a}\right|^{2}}{\partial x_{l}}=-\int_{\mathbb{R}^{2}} \frac{1}{2}\left[\frac{\alpha_{a}^{4} \Omega^{2} x_{l}}{2}+\alpha_{a}^{2} \frac{\partial V_{\Omega}\left(\alpha_{a}\left(x+y_{0}\right)\right)}{\partial x_{l}}\right] R_{j, a}^{2},
$$

where $\left(x_{1}, x_{2}\right)=x \in \mathbb{R}^{2}$. We then derive from (4.6) that for $l=1,2$,

$$
\begin{aligned}
& \int_{\mathbb{R}^{2}} \frac{1}{2}\left[\frac{\alpha_{a}^{4} \Omega^{2} x_{l}}{2}+\alpha_{a}^{2} \frac{\partial V_{\Omega}\left(\alpha_{a}\left(x+y_{0}\right)\right)}{\partial x_{l}}\right] R_{j, a}^{2} \\
= & -\int_{\mathbb{R}^{2}} \alpha_{a}^{2} \Omega\left(x^{\perp} \cdot \nabla I_{j, a}\right) \frac{\partial R_{j, a}(x)}{\partial x_{l}}-\int_{\mathbb{R}^{2}} \frac{a}{2}\left|I_{j, a}\right|^{2} \frac{\partial\left|R_{j, a}\right|^{2}}{\partial x_{l}} d x, \quad j=1,2 .
\end{aligned}
$$

Following the above identity $(E)_{j}$ for $j=1,2$, we calculate

$$
\frac{(E)_{2}-(E)_{1}}{\left\|\tilde{u}_{2, a}(x)-\tilde{u}_{1, a}(x)\right\|_{L^{\infty}\left(\mathbb{R}^{2}\right)}}
$$

which then yields from (3.1) and (3.7) that

$$
\begin{aligned}
& \int_{\mathbb{R}^{2}} \frac{\alpha_{a}^{2}}{2} \frac{\partial V_{\Omega}\left(\alpha_{a}\left(x+y_{0}\right)\right)}{\partial x_{l}}\left(R_{2, a}+R_{1, a}\right) \eta_{1, a} \\
= & -\int_{\mathbb{R}^{2}} \frac{\alpha_{a}^{4} \Omega^{2} x_{l}}{4}\left(R_{2, a}+R_{1, a}\right) \eta_{1, a}-\int_{\mathbb{R}^{2}}\left\{\alpha_{a}^{2} \Omega\left(x^{\perp} \cdot \nabla \eta_{2, a}\right) \frac{\partial R_{2, a}(x)}{\partial x_{l}}+\alpha_{a}^{2} \Omega\left(x^{\perp} \cdot \nabla I_{1, a}\right) \frac{\partial \eta_{1, a}}{\partial x_{l}}\right\} \\
& -\int_{\mathbb{R}^{2}} \frac{a}{2} \eta_{2, a}\left(I_{1, a}+I_{2, a}\right) \frac{\partial\left|R_{2, a}\right|^{2}}{\partial x_{l}}-\int_{\mathbb{R}^{2}} \frac{a}{2}\left|I_{1, a}\right|^{2} \frac{\partial\left[\eta_{1, a}\left(R_{1, a}+R_{2, a}\right)\right]}{\partial x_{l}} .
\end{aligned}
$$


We next prove (4.5) by estimating all terms of (4.8).

Following Lemma 3.3, we get that as $a \nearrow a^{*}$,

$$
\begin{aligned}
& -\int_{\mathbb{R}^{2}} \frac{\alpha_{a}^{4} \Omega^{2} x_{l}}{4}\left(R_{2, a}+R_{1, a}\right) \eta_{1, a} d x \\
= & -\frac{\alpha_{a}^{4} \Omega^{2}}{2 \sqrt{a^{*}}} \int_{\mathbb{R}^{2}} x_{l} w\left[b_{0}(w+x \cdot \nabla w)+\sum_{j=1}^{2} b_{j} \frac{\partial w}{\partial x_{j}}\right] d x+o\left(\alpha_{a}^{4}\right) \\
= & -\frac{\alpha_{a}^{4} \Omega^{2}}{4 \sqrt{a^{*}}} \int_{\mathbb{R}^{2}} b_{l} x_{l} \frac{\partial w^{2}}{\partial x_{l}} d x+o\left(\alpha_{a}^{4}\right) \\
= & \frac{\sqrt{a^{*}} \Omega^{2} b_{l} \alpha_{a}^{4}}{4}+o\left(\alpha_{a}^{4}\right), \quad l=1,2 .
\end{aligned}
$$

Similarly, we derive from Proposition 3.4 that as $a \nearrow a^{*}$,

$$
\begin{aligned}
& -\alpha_{a}^{2} \Omega \int_{\mathbb{R}^{2}} x^{\perp} \cdot \nabla \eta_{2, a}(x) \frac{\partial R_{2, a}(x)}{\partial x_{l}} d x \\
= & \frac{\Omega^{2} \alpha_{a}^{4}}{2 \sqrt{a^{*}}} \int_{\mathbb{R}^{2}} x^{\perp} \cdot \nabla\left[\left(b_{1} x_{2}-b_{2} x_{1}\right) w(x)\right] \frac{\partial w(x)}{\partial x_{l}} d x+o\left(\alpha_{a}^{4}\right) \\
= & \frac{\Omega^{2} \alpha_{a}^{4}}{2 \sqrt{a^{*}}} \int_{\mathbb{R}^{2}}\left[\left(b_{1} x_{1}+b_{2} x_{2}\right) w(x)\right] \frac{\partial w(x)}{\partial x_{l}} d x+o\left(\alpha_{a}^{4}\right) \\
= & -\frac{\sqrt{a^{*}} \Omega^{2} b_{l} \alpha_{a}^{4}}{4}+o\left(\alpha_{a}^{4}\right), \quad l=1,2 .
\end{aligned}
$$

Applying Lemma 3.1 and Proposition 3.4 we also deduce that as $a \nearrow a^{*}$,

$$
\int_{\mathbb{R}^{2}} \alpha_{a}^{2} \Omega\left(x^{\perp} \cdot \nabla I_{1, a}\right) \frac{\partial \eta_{1, a}}{\partial x_{l}} d x=o\left(\alpha_{a}^{4}\right),
$$

and

$$
\int_{\mathbb{R}^{2}} \frac{a}{2} \eta_{2, a}\left(I_{1, a}+I_{2, a}\right) \frac{\partial\left|R_{2, a}\right|^{2}}{\partial x_{l}} d x=o\left(\alpha_{a}^{4}\right), \quad \int_{\mathbb{R}^{2}} \frac{a}{2}\left|I_{1, a}\right|^{2} \frac{\partial\left[\eta_{1, a}\left(R_{1, a}+R_{2, a}\right)\right]}{\partial x_{l}} d x=o\left(\alpha_{a}^{4}\right) .
$$

Under the assumption $(V)$, we finally conclude from (4.8)-(4.12) that as $a \nearrow a^{*}$,

$$
\begin{aligned}
o\left(\alpha_{a}^{4}\right) & =\int_{\mathbb{R}^{2}} \frac{\alpha_{a}^{2}}{2} \frac{\partial V_{\Omega}\left(\alpha_{a}\left(x+y_{0}\right)\right)}{\partial x_{l}}\left(R_{2, a}+R_{1, a}\right) \eta_{1, a} d x \\
& =\frac{\alpha_{a}^{2+p}}{2} \int_{\mathbb{R}^{2}} \frac{\partial h\left(x+y_{0}\right)}{\partial x_{l}}\left(R_{2, a}+R_{1, a}\right) \eta_{1, a} d x+o\left(\alpha_{a}^{2+p}\right) \\
& =\frac{\alpha_{a}^{2+p}}{\sqrt{a^{*}}} \int_{\mathbb{R}^{2}} \frac{\partial h\left(x+y_{0}\right)}{\partial x_{l}} w\left[b_{0}(w+x \cdot \nabla w)+\sum_{j=1}^{2} b_{j} \frac{\partial w}{\partial x_{j}}\right] d x+o\left(\alpha_{a}^{2+p}\right) \\
& =\frac{\alpha_{a}^{2+p}}{2 \sqrt{a^{*}}} \int_{\mathbb{R}^{2}} \frac{\partial h\left(x+y_{0}\right)}{\partial x_{l}}\left[b_{0}\left(x \cdot \nabla w^{2}\right)+\sum_{j=1}^{2} b_{j} \frac{\partial w^{2}}{\partial x_{j}}\right] d x+o\left(\alpha_{a}^{2+p}\right), \quad l=1,2,
\end{aligned}
$$

where we have used the fact that $y_{0}$ is the unique critical point of $H(y)$. This further implies that the claim (4.5) holds true.

Step 2. The constant $b_{0}=0$ in (3.25).

Multiplying the equation (4.3) by $\left(x \cdot \nabla R_{j, a}\right)$, we have for $j=1,2$,

$$
\begin{aligned}
& -\Delta R_{j, a}\left(x \cdot \nabla R_{j, a}\right)-\alpha_{a}^{2} \Omega\left(x^{\perp} \cdot \nabla I_{j, a}\right)\left(x \cdot \nabla R_{j, a}\right) \\
= & {\left[\alpha_{a}^{2} \mu_{j, a}-\frac{\alpha_{a}^{4} \Omega^{2}|x|^{4}}{4}-\alpha_{a}^{2} V_{\Omega}\left(\alpha_{a}\left(x+y_{0}\right)\right)\right] R_{j, a}\left(x \cdot \nabla R_{j, a}\right) } \\
& +a\left|\tilde{u}_{j, a}\right|^{2} R_{j, a}\left(x \cdot \nabla R_{j, a}\right) \quad \text { in } \mathbb{R}^{2} .
\end{aligned}
$$


Using the integration by parts, we note from (2.24) that for $j=1,2$,

$$
\begin{gathered}
A_{j, a}:=-\int_{\mathbb{R}^{2}} \Delta R_{j, a}\left(x \cdot \nabla R_{j, a}\right) d x \\
=-\lim _{R \rightarrow \infty} \int_{\partial B_{R}(0)}\left[\frac{\partial R_{j, a}}{\partial \nu}\left(x \cdot \nabla R_{j, a}\right)-(x \cdot \nu)\left|\nabla \hat{R}_{j, a}\right|^{2}\right] d S \\
=0 \\
B_{j, a}:=\int_{\mathbb{R}^{2}}\left[\alpha_{a}^{2} \mu_{j, a}-\frac{\alpha_{a}^{4} \Omega^{2}|x|^{2}}{4}-\alpha_{a}^{2} V_{\Omega}\left(\alpha_{a}\left(x+y_{0}\right)\right)\right] R_{j, a}\left(x \cdot \nabla R_{j, a}\right) \\
=-\int_{\mathbb{R}^{2}} R_{j, a}^{2}\left[\alpha_{a}^{2} \mu_{j, a}-\frac{\alpha_{a}^{4} \Omega^{2}|x|^{2}}{2}-\alpha_{a}^{2} V_{\Omega}\left(\alpha_{a}\left(x+y_{0}\right)\right)-\frac{\alpha_{a}^{2}}{2} x \cdot \nabla_{x} V_{\Omega}\left(\alpha_{a}\left(x+y_{0}\right)\right)\right],
\end{gathered}
$$

and

$$
\begin{aligned}
C_{j, a}: & =a \int_{\mathbb{R}^{2}}\left|\tilde{u}_{j, a}\right|^{2} R_{j, a}\left(x \cdot \nabla R_{j, a}\right) \\
& =\frac{a}{4} \int_{\mathbb{R}^{2}}\left(x \cdot \nabla R_{j, a}^{4}\right)+\frac{a}{2} \int_{\mathbb{R}^{2}} I_{j, a}^{2}\left(x \cdot \nabla R_{j, a}^{2}\right) \\
& =-\frac{a}{2} \int_{\mathbb{R}^{2}} R_{j, a}^{4}+\frac{a}{2} \int_{\mathbb{R}^{2}} I_{j, a}^{2}\left(x \cdot \nabla R_{j, a}^{2}\right) .
\end{aligned}
$$

Therefore, we derive from above that

$$
\frac{\left(D_{2, a}-D_{1, a}\right)}{\left\|\tilde{u}_{2, a}-\tilde{u}_{1, a}\right\|_{L^{\infty}\left(\mathbb{R}^{2}\right)}}=\frac{\left(B_{2, a}-B_{1, a}\right)+\left(C_{2, a}-C_{1, a}\right)}{\left\|\tilde{u}_{2, a}-\tilde{u}_{1, a}\right\|_{L^{\infty}\left(\mathbb{R}^{2}\right)}},
$$

where $D_{j, a}$ is defined by

$$
D_{j, a}:=-\int_{\mathbb{R}^{2}} \alpha_{a}^{2} \Omega\left(x^{\perp} \cdot \nabla I_{j, a}\right)\left(x \cdot \nabla R_{j, a}\right), \quad j=1,2 .
$$

We next estimate the terms containing $D_{j, a}$ and $B_{j, a}$ of (4.15) as follows.

As for the term containing $D_{j, a}$, applying Lemmas 3.1 and 3.2. we infer from Proposition 3.4 that as $a \nearrow a^{*}$,

$$
\begin{aligned}
& \frac{D_{2, a}-D_{1, a}}{\left\|\tilde{u}_{2, a}-\tilde{u}_{1, a}\right\|_{L^{\infty}\left(\mathbb{R}^{2}\right)}} \\
= & -\alpha_{a}^{2} \int_{\mathbb{R}^{2}} \Omega\left(x^{\perp} \cdot \nabla \eta_{2, a}\right)\left(x \cdot \nabla R_{2, a}\right)-\alpha_{a}^{2} \int_{\mathbb{R}^{2}} \Omega\left(x^{\perp} \cdot \nabla I_{1, a}\right)\left(x \cdot \nabla \eta_{1, a}\right) \\
= & -\alpha_{a}^{2} \int_{\mathbb{R}^{2}} \Omega\left(x^{\perp} \cdot \nabla \eta_{2, a}\right)\left[x \cdot\left(\nabla \frac{w}{\sqrt{a^{*}}}\right)+x \cdot \nabla\left(R_{2, a}-\frac{w}{\sqrt{a^{*}}}\right)\right]+o\left(\alpha_{a}^{4}\right) \\
= & \alpha_{a}^{2} \frac{\Omega}{\sqrt{a^{*}}} \int_{\mathbb{R}^{2}}\left[x^{\perp} \cdot \nabla(x \cdot \nabla w)\right] \eta_{2, a}+o\left(\alpha_{a}^{4}\right)=o\left(\alpha_{a}^{4}\right),
\end{aligned}
$$

where (3.1) and (3.7) are also used. As for the term containing $B_{j, a}$, we obtain from the assumption $(V)$ that as $a \nearrow a^{*}$,

$$
\begin{aligned}
& \frac{B_{2, a}-B_{1, a}}{\left\|\tilde{u}_{2, a}-\tilde{u}_{1, a}\right\|_{L^{\infty}}} \\
= & \int_{\mathbb{R}^{2}}\left[\frac{\alpha_{a}^{4} \Omega^{2}|x|^{2}}{2}+\alpha_{a}^{2} V_{\Omega}\left(\alpha_{a}\left(x+y_{0}\right)\right)+\frac{\alpha_{a}^{2}}{2}\left(x+y_{0}\right) \cdot \nabla_{x} V_{\Omega}\left(\alpha_{a}\left(x+y_{0}\right)\right)\right] \\
& \cdot\left(R_{1, a}+R_{2, a}\right) \eta_{1, a} d x-J_{a}-K_{a}, \\
= & \int_{\mathbb{R}^{2}}\left[\frac{\alpha_{a}^{4} \Omega^{2}|x|^{2}}{2}+\frac{2+p}{2} \alpha_{a}^{2+p} h\left(x+y_{0}\right)\right]\left(R_{1, a}+R_{2, a}\right) \eta_{1, a} d x-J_{a}-K_{a}+o\left(\alpha_{a}^{2+p}\right),
\end{aligned}
$$


where the fact $x \cdot \nabla h(x)=p h(x)$ is used in the last equality. Here the terms $J_{a}$ and $K_{a}$ are defined by

$$
J_{a}:=\frac{\alpha_{a}^{2}}{2} \int_{\mathbb{R}^{2}}\left[y_{0} \cdot \nabla_{x} V_{\Omega}\left(\alpha_{a}\left(x+y_{0}\right)\right)\right]\left(R_{1, a}+R_{2, a}\right) \eta_{1, a} d x,
$$

and

$$
K_{a}:=\alpha_{a}^{2} \int_{\mathbb{R}^{2}} \frac{R_{2, a}^{2} \mu_{2, a}-R_{1, a}^{2} \mu_{1, a}}{\left\|\tilde{u}_{2, a}-\tilde{u}_{1, a}\right\|_{L^{\infty}\left(\mathbb{R}^{2}\right)}} d x .
$$

Applying (2.19), one can note from the first identity of (4.13) that as $a \nearrow a^{*}$,

$$
J_{a}=o\left(\alpha_{a}^{4}\right)
$$

Using Lemma 3.1 and Proposition 3.4, we derive from (4.4) that $K_{a}$ satisfies

$$
\begin{aligned}
K_{a}:= & \alpha_{a}^{2} \int_{\mathbb{R}^{2}} \frac{\left|\tilde{u}_{2, a}\right|^{2} \mu_{2, a}-\left|\tilde{u}_{1, a}\right|^{2} \mu_{1, a}}{\left\|\tilde{u}_{2, a}-\tilde{u}_{1, a}\right\|_{L^{\infty}\left(\mathbb{R}^{2}\right)}}-\alpha_{a}^{2} \int_{\mathbb{R}^{2}} \frac{I_{2, a}^{2} \mu_{2, a}-I_{1, a}^{2} \mu_{1, a}}{\left\|\tilde{u}_{2, a}-\tilde{u}_{1, a}\right\|_{L^{\infty}\left(\mathbb{R}^{2}\right)}} \\
= & \frac{\alpha_{a}^{2}\left(\mu_{2, a}-\mu_{1, a}\right)}{\left\|\tilde{u}_{2, a}-\tilde{u}_{1, a}\right\|_{L^{\infty}\left(\mathbb{R}^{2}\right)}}-\alpha_{a}^{2} \int_{\mathbb{R}^{2}} \eta_{2, a}\left(I_{1, a}+I_{2, a}\right) \mu_{2, a} \\
& -\alpha_{a}^{2} \frac{\mu_{2, a}-\mu_{1, a}}{\left\|\tilde{u}_{2, a}-\tilde{u}_{1, a}\right\|_{L^{\infty}\left(\mathbb{R}^{2}\right)}} \int_{\mathbb{R}^{2}} I_{1, a}^{2} \\
= & \frac{\alpha_{a}^{2}\left(\mu_{2, a}-\mu_{1, a}\right)}{\left\|\tilde{u}_{2, a}-\tilde{u}_{1, a}\right\|_{L^{\infty}\left(\mathbb{R}^{2}\right)}}+o\left(\alpha_{a}^{4}\right) \text { as } a \nearrow a^{*} .
\end{aligned}
$$

It then follows from above that

$$
\begin{aligned}
& \frac{B_{2, a}-B_{1, a}}{\left\|\tilde{u}_{2, a}-\tilde{u}_{1, a}\right\|_{L^{\infty}\left(\mathbb{R}^{2}\right)}} \\
= & \int_{\mathbb{R}^{2}}\left[\frac{\alpha_{a}^{4} \Omega^{2}|x|^{2}}{2}+\frac{2+p}{2} \alpha_{a}^{2+p} h\left(x+y_{0}\right)\right]\left(R_{1, a}+R_{2, a}\right) \eta_{1, a} \\
& -\frac{\alpha_{a}^{2}\left(\mu_{2, a}-\mu_{1, a}\right)}{\left\|\hat{u}_{2, a}-\hat{u}_{1, a}\right\|_{L^{\infty}\left(\mathbb{R}^{2}\right)}}+o\left(\alpha_{a}^{2+p}\right) \text { as } a \nearrow a^{*} .
\end{aligned}
$$

Applying Lemma 3.1 and Proposition 3.4 we obtain that as $a \nearrow a^{*}$,

$$
\begin{aligned}
& \frac{C_{2, a}-C_{1, a}}{\left\|\tilde{u}_{2, a}-\tilde{u}_{1, a}\right\|_{L^{\infty}\left(\mathbb{R}^{2}\right)}} \\
= & -\frac{a}{2} \int_{\mathbb{R}^{2}} \frac{\left|\tilde{u}_{2, a}\right|^{4}-\left|\tilde{u}_{1, a}\right|^{4}}{\left\|\tilde{u}_{2, a}-\tilde{u}_{1, a}\right\|_{L^{\infty}\left(\mathbb{R}^{2}\right)}}+\frac{a}{2} \int_{\mathbb{R}^{2}} \frac{\left(\left|I_{2, a}\right|^{4}-\left|I_{1, a}\right|^{4}\right)+2\left(\left|R_{2, a}\right|^{2}\left|I_{2, a}\right|^{2}-\left|R_{1, a}\right|^{2}\left|I_{1, a}\right|^{2}\right)}{\left\|\tilde{u}_{2, a}-\tilde{u}_{1, a}\right\|_{L^{\infty}\left(\mathbb{R}^{2}\right)}} \\
& +\frac{a}{2} \int_{\mathbb{R}^{2}} I_{2, a}^{2}\left[x \cdot \nabla\left[\eta_{1, a}\left(R_{1, a}+R_{2, a}\right)\right]\right]+\frac{a}{2} \int_{\mathbb{R}^{2}} \eta_{2, a}\left(I_{1, a}+I_{2, a}\right)\left(x \cdot \nabla R_{1, a}^{2}\right) \\
= & -\frac{a}{2} \int_{\mathbb{R}^{2}} \frac{\left|\tilde{u}_{2, a}\right|^{4}-\left|\tilde{u}_{1, a}\right|^{4}}{\left\|\tilde{u}_{2, a}-\tilde{u}_{1, a}\right\|_{L^{\infty}\left(\mathbb{R}^{2}\right)}}+o\left(\alpha_{a}^{4}\right) .
\end{aligned}
$$

Note from (4.2) that

$$
-\alpha_{a}^{2} \frac{\mu_{2, a}-\mu_{1, a}}{\left\|\tilde{u}_{2, a}-\tilde{u}_{1, a}\right\|_{L^{\infty}\left(\mathbb{R}^{2}\right)}}-\frac{a}{2} \int_{\mathbb{R}^{2}} \frac{\left|\tilde{u}_{2, a}\right|^{4}-\left|\tilde{u}_{1, a}\right|^{4}}{\left\|\tilde{u}_{2, a}-\tilde{u}_{1, a}\right\|_{L^{\infty}\left(\mathbb{R}^{2}\right)}} d x \equiv 0 .
$$

Following this identity, we then conclude from (4.15)-(4.18) that as $a \nearrow a^{*}$,

$$
\begin{aligned}
o\left(\alpha_{a}^{4}\right) & =\frac{\left(B_{2, a}-B_{1, a}\right)+\left(C_{2, a}-C_{1, a}\right)}{\left\|\tilde{u}_{2, a}-\tilde{u}_{1, a}\right\|_{L^{\infty}\left(\mathbb{R}^{2}\right)}} \\
& =\int_{\mathbb{R}^{2}}\left[\frac{\Omega^{2} \alpha_{a}^{4}|x|^{2}}{2}+\frac{(2+p) \alpha_{a}^{2+p}}{2} h\left(x+y_{0}\right)\right]\left(R_{1, a}+R_{2, a}\right) \eta_{1, a} d x+o\left(\alpha_{a}^{2+p}\right) \\
& =\frac{(2+p) \alpha_{a}^{2+p}}{\sqrt{a^{*}}} \int_{\mathbb{R}^{2}} h\left(x+y_{0}\right) w \eta_{1} d x+\frac{\Omega^{2} \alpha_{a}^{4}}{\sqrt{a^{*}}} \int_{\mathbb{R}^{2}}|x|^{2} w \eta_{1} d x+o\left(\alpha_{a}^{2+p}\right) .
\end{aligned}
$$


Since $y_{0}$ is the unique critical point of $H(y)$, we finally derive from (4.19) that if $1<p<2$,

$$
\begin{aligned}
0 & =\int_{\mathbb{R}^{2}} h\left(x+y_{0}\right) w \eta_{1} d x \\
& =b_{0} \int_{\mathbb{R}^{2}} h\left(x+y_{0}\right) w(w+x \cdot \nabla w) d x+\sum_{i=1}^{2} b_{i} \int_{\mathbb{R}^{2}} \frac{1}{2} h\left(x+y_{0}\right) \frac{\partial_{i} w^{2}}{\partial x_{i}} d x \\
& =b_{0} \int_{\mathbb{R}^{2}} h\left(x+y_{0}\right) w^{2} d x-\frac{b_{0}}{2} \int_{\mathbb{R}^{2}}\left(x \cdot \nabla h\left(x+y_{0}\right) w^{2}+2 h\left(x+y_{0}\right) w^{2}\right) d x \\
& =-\frac{b_{0}}{2} \int_{\mathbb{R}^{2}}\left(\left(x+y_{0}\right) \cdot \nabla h\left(x+y_{0}\right) w^{2}\right) d x \\
& =-\frac{p b_{0}}{2} \int_{\mathbb{R}^{2}} h\left(x+y_{0}\right) w^{2} d x
\end{aligned}
$$

which further implies that $b_{0}=0$. Similarly, the above conclusion also holds true in the case $p=2$, and the claim $b_{0}=0$ is therefore proved.

Step 3. The constants $b_{1}=b_{2}=0$.

By Step 2, we deduce from (4.5) that

$$
\sum_{j=1}^{2} b_{j} \int_{\mathbb{R}^{2}} \frac{\partial h\left(x+y_{0}\right)}{\partial x_{l}} \frac{\partial w^{2}}{\partial x_{j}} d x=0, \quad l=1,2 .
$$

Using the non-degeneracy assumption of the critical point $y_{0}$, we then derive from (4.20) that $b_{1}=b_{2}=0$, and the proof of Step 3 is therefore complete.

By the exponential decay of Lemma 3.3 we obtain that $\eta_{a} \rightarrow \eta_{0}=\eta_{1}+i \eta_{2} \not \equiv 0$ uniformly in $C^{1}\left(\mathbb{R}^{2}\right)$ as $a \nearrow a^{*}$, due to the fact that $\left\|\eta_{a}\right\|_{L^{\infty}} \equiv 1$. However, we conclude from Step 2 and Step 3 that $\eta_{0} \equiv 0$, a contradiction. This completes the proof of Theorem 1.1 .

Acknowledgements: The authors are very grateful to the referee for many valuable suggestions which lead to the great improvements of the present paper.

\section{References}

[1] J. R. Abo-Shaeer, C. Raman, J. M. Vogels and W. Ketterle, Observation of vortex lattices in Bose-Einstein condensate, Science 292 (2001), 476.

[2] A. Aftalion, Vortices in Bose-Einstein condensates, Progress in Nonlinear Differential Equations and their Applications, 67. Birkhäuser Boston, Inc., Boston, MA, 2006.

[3] A. Aftalion, Vortex patterns in Bose Einstein condensates, Perspectives in nonlinear partial differential equations, Contemp. Math. 446, Amer. Math. Soc., Providence, RI (2007), 1-18.

[4] M. H. Anderson, J. R. Ensher, M. R. Matthews, C. E. Wieman and E. A. Cornell, Observation of Bose-Einstein condensation in a dilute atomic vapor, Science 269 (1995), 198-201.

[5] J. Arbunich, I. Nenciu and C. Sparber, Stability and instability properties of rotating Bose-Einstein condensates, Lett. Math. Phys. 109 (2019), 1415-1432.

[6] G. Arioli and A. Szulkin, A semilinear Schrödinger equation in the presence of a magnetic field, Arch. Ration. Mech. Anal. 170 (2003), 277-295. 
[7] W. Bao and Y. Cai, Ground states of two-component Bose-Einstein condensates with an internal atomic Josephson junction, East Asia J. Appl. Math. 1 (2011), 49-81.

[8] T. Bartsch, E. N. Dancer and S. Peng. On multi-bump semi-classical bound states of nonlinear Schrödinger equations with electromagnetic fields, Adv. Differential Equations 11 (2006), 781-812.

[9] I. Bloch, J. Dalibard and W. Zwerger, Many-body physics with ultracold gases, Reviews of Modern Phys. 80 (2008), 885-964.

[10] C. C. Bradley, C. A. Sackett and R. G. Hulet, Bose-Einstein condensation of lithium: observation of limited condensate number, Phys. Rev. Lett. 78 (1997), 985.

[11] C. C. Bradley, C. A. Sackett, J. J. Tollett and R. G. Hulet, Evidence of Bose-Einstein condensation in an atomic gas with attractive interactions, Phys. Rev. Lett. 75 (1995), 1687. Erratum Phys. Rev. Lett. 79 (1997), 1170.

[12] D. Cao, S. Li and P. Luo, Uniqueness of positive bound states with multibump for nonlinear Schrödinger equations, Calc. Var. Partial Differential Equations 54 (2015), 4037-4063.

[13] D. Cao and Z. Tang, Existence and uniqueness of multi-bump bound states of nonlinear Schrödinger equations with electromagnetic fields, J. Differential Equations 222 (2006), $381-424$.

[14] L. D. Carr and C. W. Clark, Vortices in attractive Bose-Einstein condensates in two dimensions, Phys. Rev. Lett. 97 (2006), 010403.

[15] T. Cazenave, Semilinear Schrödinger equations, Courant Lecture Notes in Mathematics Vol. 10, Courant Institute of Mathematical Science/AMS, New York, 2003.

[16] T. Cazenave and P. L. Lions, Orbital stability of standing waves for some nonlinear Schrödinger equations, Comm. Math. Phys. 85 (1982), no. 4, 549-561.

[17] N. R. Cooper, Rapidly rotating atomic gases, Adv. Phys. 57 (2008), 539-616.

[18] M. Correggi and N. Rougerie, Boundary behavior of the Ginzburg-Landau order parameter in the surface superconductivity regime, Arch. Rational Mech. Anal. 219 (2016), 553-606.

[19] M. Correggi, N. Rougerie and J. Yngvason, The transition to a giant vortex phase in a fast rotating Bose-Einstein condensate, Comm. Math. Phys. 303 (2011), 451-508.

[20] F. Dalfovo, S. Giorgini, L. P. Pitaevskii and S. Stringari, Theory of Bose-Einstein condensation in trapped gases, Reviews of Modern Phys. 71 (1999), 463-512.

[21] Y. Deng, C. Lin and S. Yan, On the prescribed scalar curvature problem in $\mathbb{R}^{N}$, local uniqueness and periodicity, J. Math. Pures Appl. 104 (2015), 1013-1044.

[22] M. J. Esteban and P. L. Lions, Stationary solutions of nonlinear Schrödinger equations with an external magnetic field, Partial differential equations and the calculus of variations, Vol. I, 401-449, Progr. Nonlinear Differential Equations Appl. 1, Birkhuser Boston, Boston, MA, 1989.

[23] A. L. Fetter, Rotating trapped Bose-Einstein condensates, Reviews of Modern Phys. 81 (2009), 647-691.

[24] R. L. Frank, Ground states of semi-linear PDEs, Lecture notes from summer school on "Current Topics in Mathematical Physics", CIRM Marseille, 2013.

[25] B. Gidas, W. Ni and L. Nirenberg, Symmetry of positive solutions of nonlinear elliptic equations in $\mathbb{R}^{n}$, Mathematical analysis and applications Part A, Adv. in Math. Suppl. Stud. Vol. 7, Academic Press, New York (1981), 369-402. 
[26] D. Gilbarg and N. S. Trudinger, Elliptic Partial Differential Equations of Second Order, Springer, 1997.

[27] M. Grossi, On the number of single-peak solutions of the nonlinear Schrödinger equations, Ann. Inst. H. Poincaré Anal. Non Linéaire 19 (2002), 261-280.

[28] Y. Guo, S. Li, J. Wei and X. Zeng, Ground states of two-component attractive BoseEinstein condensates II: semi-trivial limit behavior, Trans. Amer. Math. Soc. 371 (2019), 6903-6948.

[29] Y. Guo, C. Lin and J. Wei, Local uniqueness and refined spike profiles of ground states for two-dimensional attractive Bose-Einstein condensates, SIAM J. Math. Anal. 49 (2017), 3671-3715.

[30] Y. Guo, Y. Luo and W. Yang, The nonexistence of vortices for rotating Bose-Einstein condensates with attractive interactions, Arch. Rational Mech. Anal. 238 (2020), 12311281.

[31] Y. Guo and R. Seiringer, On the mass concentration for Bose-Einstein condensates with attractive interactions, Lett. Math. Phys. 104 (2014), 141-156.

[32] Y. Guo, Z. Wang, X. Zeng and H. Zhou, Properties of ground states of attractive GrossPitaevskii equations with multi-well potentials, Nonlinearity 31 (2018), 957-979.

[33] Y. Guo, X. Zeng and H. Zhou, Energy estimates and symmetry breaking in attractive Bose-Einstein condensates with ring-shaped potentials, Ann. Inst. H. Poincaré Anal. Non Linéaire 33 (2016), 809-828.

[34] Q. Han and F. Lin, Elliptic Partial Differential Equations, Courant Lecture Note in Math. 1, Courant Institute of Mathematical Science/AMS, New York, 2011.

[35] C. Huepe, S. Metens, G. Dewel, P. Borckmans and M.E. Brachet, Decay rates in attractive Bose-Einstein condensates, Phys. Rev. Lett. 82 (1999), 1616-1619.

[36] R. Ignat and V. Millot, The critical velocity for vortex existence in a two-dimensional rotating Bose-Einstein condensate, J. Funct. Anal. 233 (2006), 260-306.

[37] R. Ignat and V. Millot, Energy expansion and vortex location for a two-dimensional rotating Bose-Einstein condensate, Rev. Math. Phys. 18 (2006), 119-162.

[38] Y. Kagan, A. E. Muryshev and G.V. Shlyapnikov, Collapse and Bose-Einstein condensation in a trapped Bose gas with nagative scattering length, Phys. Rev. Lett. 81 (1998), 933-937.

[39] K. Kasamatsu, M. Tsubota and M. Ueda, Giant hole and circular superflow in a fast rotating Bose-Einstein condensate, Phys. Rev. B 66 (2002), 053606.

[40] M. K. Kwong, Uniqueness of positive solutions of $\Delta u-u+u^{p}=0$ in $\mathbb{R}^{N}$, Arch. Rational Mech. Anal. 105 (1989), 243-266.

[41] M. Lewin, P. T. Nam and N. Rougerie, A note on 2D focusing many-boson systems, Proc. Amer. Math. Soc. 145 (2017), 2441-2454.

[42] M. Lewin, P. T. Nam and N. Rougerie, Blow-up profile of rotating 2D focusing Bose gases, Macroscopic Limits of Quantum Systems, a conference in honor of Herbert Spohn's 70th birthday, Springer Verlag, 2018, 145-170.

[43] G. Li, S. Peng and C. Wang, Infinitely many solutions for nonlinear Schrödinger equations with electromagnetic fields, J. Differential Equations 251 (2011), 3500-3521. 
[44] E. H. Lieb and M. Loss, Analysis, Graduate Studies in Mathematics Vol. 14. Amer. Math. Soc., Providence, RI, second edition, 2001.

[45] E. H. Lieb and R. Seiringer, Derivation of the Gross-Pitaevskii equation for rotating Bose gases, Comm. Math. Phys. 264 (2006), 505-537.

[46] E. H. Lieb, R. Seiringer, J. P. Solovej and J. Yngvason, The mathematics of the Bose gas and its condensation, Oberwolfach Seminars, Birkhäuser Verlag, Basel, 2005.

[47] E. H. Lieb, R. Seiringer and J. Yngvason, Bosons in a trap: A rigorous derivation of the Gross-Pitaevskii energy functional, Phys. Rev. A 61 (2000), 043602.

[48] E. Lundh, A. Collin and K.-A. Suominen, Rotational states of Bose gases with attractive interactions in anharmonic traps, Phys. Rev. Lett. 92 (2004), 070401.

[49] P. Luo, S. Peng and S. Yan, Excited states on Bose-Einstein condensates with attractive interactions, submitted, (2019), arXiv:1909.08828.

[50] K. Madison, F. Chevy, J. Dalibard and W. Wohlleben, Vortex formation in a stirred Bose-Einstein condensate, Phys. Rev. Lett. 84 (2000), 806.

[51] K. Madison, F. Chevy, J. Dalibard and W. Wohlleben, Vortices in a stirred Bose-Einstein condensate, J. Mod. Opt. 47 (2000), 2715-2723.

[52] P. T. Nam and N. Rougerie, Improved stability for $2 D$ attractive Bose gases, J. Math. Phys. 61 (2020), 021901.

[53] W. Ni and I. Takagi, On the shape of least-energy solutions to a semilinear Neumann problem, Comm. Pure Appl. Math. 44 (1991), 819-851.

[54] N. Rougerie, Scaling limits of bosonic ground states, from many-body to nonlinear Schrödinger, EMS Surveys in Math. Sciences, EMS (2021), in press, arXiv: 2002.02678.

[55] C. A. Sackett, H. T. C. Stoof and R. G. Hulet, Growth and collapse of a Bose-Einstein condensate with attractive interactions, Phys. Rev. Lett. 80 (1998), 2031.

[56] M. I. Weinstein, Nonlinear Schrödinger equations and sharp interpolations estimates, Comm. Math. Phys. 87 (1983), 567-576.

[57] N. K. Wilkin, J. M. F. Gunn, and R. A. Smith, Do attractive Bosons condense?, Phys. Rev. Lett. 80 (1998), 2265.

[58] J. Zhang, Stability of attractive Bose-Einstein condensates, J. Stat. Phys. 101 (2000), $731-746$.

[59] M. W. Zwierlein, J. R. Abo-Shaeer, A. Schirotzek, C. H. Schunck and W. Ketterle, Vortices and superfluidity in a strongly interacting fermi gas, Nature 435 (2005), 10471051. 